\title{
A Study of Detonation Propagation and Diffraction with Compliant Confinement
}

J.W. Banks, D.W. Schwendeman, A.K. Kapila, W.D. Henshaw

August 16, 2007

Combustion Theory and Modeling 
This document was prepared as an account of work sponsored by an agency of the United States Government. Neither the United States Government nor the University of California nor any of their employees, makes any warranty, express or implied, or assumes any legal liability or responsibility for the accuracy, completeness, or usefulness of any information, apparatus, product, or process disclosed, or represents that its use would not infringe privately owned rights. Reference herein to any specific commercial product, process, or service by trade name, trademark, manufacturer, or otherwise, does not necessarily constitute or imply its endorsement, recommendation, or favoring by the United States Government or the University of California. The views and opinions of authors expressed herein do not necessarily state or reflect those of the United States Government or the University of California, and shall not be used for advertising or product endorsement purposes. 


\title{
A Study of Detonation Propagation and Diffraction with Compliant Confinement
}

\author{
J.W. Banks \\ Computational Sciences Research Institute, Sandia National Lab, \\ Albuquerque, New Mexico, 87185-1322 \\ D.W. Schwendeman and A.K. Kapila \\ Department of Mathematical Sciences, Rensselaer Polytechnic Institute, \\ Troy, New York, 12180 \\ W.D. Henshaw \\ Center for Applied Scientific Computing, Lawrence Livermore National Lab, \\ Livermore, California, 94551
}

August 11, 2007

\begin{abstract}
A previous computational study of diffracting detonations with the ignition-and-growth model demonstrated that contrary to experimental observations, the computed solution did not exhibit dead zones. For a rigidly confined explosive it was found that while diffraction past a sharp corner did lead to a temporary separation of the lead shock from the reaction zone, the detonation re-established itself in due course and no pockets of unreacted material were left behind. The present investigation continues to focus on the potential for detonation failure within the ignition-and-growth (IG) model, but now for a compliant confinement of the explosive. The aim of the present paper is two fold. First, in order to compute solutions of the governing equations for multi-material reactive flow, a numerical method of solution is developed and discussed. The method is a Godunov-type, fractional-step scheme which incorporates an energy correction to suppress numerical oscillations that would occur near the material interface separating the reactive material and the inert confiner for standard conservative schemes. The numerical method uses adaptive mesh refinement (AMR) on overlapping grids, and the accuracy of solutions is well tested using a two-dimensional rate-stick problem for both strong and weak inert confinements. The second aim of the paper is to extend the previous computational study of the IG model by considering two related problems. In the first problem, the corner-turning configuration is re-examined, and it is shown that in the matter of detonation failure, the absence of rigid confinement does not affect the outcome in a material way; sustained dead zones continue to elude the model. In the second problem, detonations propagating down a compliantly confined pencil-shaped configuration are computed for a variety of cone angles of the tapered section. It is found, in accord with experimental observation, that if the cone angle is small enough, the detonation fails prior to reaching the cone tip. For both the corner-turning and the pencil-shaped configurations, mechanisms underlying the behavior of the computed solutions are identified. It is concluded that disagreement between computation and experiment in the corner-turning case lies in the absence, in the model, of a mechanism that allows the explosive to undergo desensitization when subjected to a weak shock.
\end{abstract}

\section{Introduction}

A condensed-phase, high-energy explosive (HE) is a granular aggregate consisting of crystalline fragments of the energetic constituent held together by an inert plastic binder. Subsequent to exposure to an initiating 
stimulus such as a shock, establishment of detonation in such a material is the result of thermo-mechanical processes at the scale of the grains creating discrete hot spots, where reaction begins preferentially and then spreads to the remaining bulk. The recognition of long standing that phenomena observed at the macro scale owe their genesis to events at the grain scale has not yet resulted in an ab initio model that couples the scale of observation to the scale of driving processes in a rational manner. Standing in the way are the absence of accurate mathematical descriptions of the relevant fine-scale processes, and the lack of a theoretical and computational framework that would facilitate flow of information across scales. Both are active topics of research in a variety of fields including explosives science, and although much progress has been made, a robust, reliable model of condensed-phase explosives based on fundamental principles is yet to emerge.

As a consequence, existing continuum theories tend to be phenomenological descriptions at varying levels of sophistication. One approach favored by practitioners is represented in the ignition-and-growth (IG) model. Originally put forth by Lee and Tarver [1] and later refined by Tarver and colleagues $[2,3,4,5,6]$, it treats the explosive as a homogeneous mixture of two distinct constituents, the unreacted explosive and the products of reaction, at pressure and temperature equilibrium. To each constituent is assigned an equation of state, and a single reaction-rate law is postulated for the conversion of the explosive to products. Another approach, exemplified by Baer and Nunziato [7], treats the solid reactant and the gaseous product as two distinct phases, each with its own balance laws of mass, momentum and energy, plus a rule that allows compaction of the solid phase driven by pressure difference between the phases. Terms representing interfacial exchange of mass, momentum and energy appear, corresponding to the nonequilibrium processes of reaction, drag and heat transfer. Mathematically, these models are systems of hyperbolic partial differential equations (PDEs) that are generalizations of the Euler equations of gasdynamics.

While the two-phase model continues to undergo further theoretical scrutiny and computational development (see, for example, $[8,9]$ and the references therein), the IG model has seen wide use as a framework for simulating different classes of experiments, in a variety of configurations and for a number of explosive formulations (see, for example, [10] and the references therein). In a recent study [11] this model was subjected to a detailed theoretical and computational analysis, and descriptions of initiating transients as well as of structures of steadily propagating planar detonations were obtained. Additionally, prompted by experimental observation of dead zones (sustained pockets of unreacted material) in situations where a detonation turns a corner and undergoes diffraction, this study sought to determine whether the IG model could capture this feature. For a rigidly confined explosive, it was shown conclusively that although a temporary detachment of the reaction zone from the lead shock did occur upon diffraction, in due course the detonation re-established itself without leaving behind any pockets of unreacted material. The possible mechanisms of detonation re-initiation subsequent to the temporary and localized failure were also identified. The computations in [11], which built on the earlier work in [12], employed a Strang-type fractional-step method, where the nonlinear convective terms in the PDEs were integrated using a second-order, slope-limited extension of Godunov's method in one fractional step and the stiff reactive source terms in the equations were treated using a second-order Runge-Kutta error-control scheme in the other step. Adaptive mesh refinement (AMR) was employed to locally increase the grid resolution near detonations, shocks and contact discontinuities, and overlapping grids were used to handle complex geometry.

The present paper extends this work in two related directions, again motivated by experimental observations. First, corner turning is re-examined by relaxing the condition of rigid confinement. The intent is to explore whether deformation of the confiner in response to the high pressure behind the detonation influences the post-diffraction behavior in any material way, especially as regards to failure. Detonation diffraction at a $90^{\circ}$ corner in an expanding geometry is considered using a two-cylinder configuration consisting of a "donor" charge attached to a coaxial "acceptor" charge of a larger diameter. This geometric configuration is motivated by experiments discussed by Ferm et al in [13], and the parameters of the IG model are taken to be those for the explosive PBX 9502 [6]. A steady detonation in the donor charge is established from a high-pressure booster state, and as the detonation crosses into the acceptor charge, the main interest is in the post-diffraction behavior. The results of the experiments suggest that in the post-diffracted state the reaction zone separates from the leading shock, leading to the appearance of a low-density region of unreacted explosive, a "dead-zone." We examine the behavior numerically for strong and weak confinements, and for the strong case we also compare the results with those for a rigid confinement. While the details of the diffraction behavior differ significantly for the strong and weak confinements, the ultimate 
outcome is similar. In both cases the reaction zone separates from the leading shock temporarily, but the detonation is re-established and any unreacted explosive left behind the weakened leading shock is ultimately consumed. One explanation for the behavior seen in the numerical results is that the present IG model is not rich enough to reproduce detonation failure, as it does not account explicitly for desensitization of the heterogeneous explosive upon exposure to the weakened leading shock in the post-diffracted flow [14].

Second, the propagation and dynamic failure of a detonation in conical rate sticks is examined for a range of included cone angles. This problem is motivated by recent experiments performed by Salyer and Hill [15]. The numerical calculations employ the IG model with parameters chosen for PBX 9502, as in the corner-turning problem, and take the explosive to be weakly confined. The main focus of this problem is the behavior of the detonation as it traverses the conical section, and in particular, whether detonation failure occurs as a result of losses to the confiner overcoming the decreasing energy generated by reaction behind the leading shock. For small included cone angles, a weakening of the detonation is observed followed by a separation of the reaction zone and the leading shock before the detonation reaches the tip of the cone. For larger included cone angles, the detonation may fail very close to the tip of the cone, or it may run to the tip with no apparent weakening or separation. The behavior along the axis of symmetry is examined to judge failure, and the velocity of the detonation along the edge of the cone is determined from the numerical calculations and found to agree well with the experimental observations in [15]. This agreement suggests that unlike corner-turning, in this configuration the IG model does capture observed detonation behavior.

Solutions of the IG model for the corner-turning and cone problems require an extension of the numerical approach employed in [11] to handle the multi-material system and the interface (a contact discontinuity) separating the explosive and the confiner accurately and robustly. For multi-material flow it is well known that numerical oscillations occur in the solutions obtained by standard shock-capturing methods, such as Godunov's method, near the material interface where the state of the flow changes abruptly. Various schemes have been devised to address this difficulty, see [16, 17, 18, 19, 20] for example. In a recent work [21], we developed an accurate numerical method for nonreactive multi-material flow which can handle a wide range of equations of state for the mixture. In this method, numerical oscillations near the material interface are suppressed by incorporating an energy correction into the Godunov scheme. The essential idea behind this correction is to modify the result of the conservative Godunov step locally near the material interface so that the numerical result of an associated flow with uniform pressure and velocity would be free of numerical oscillations. In the present paper the numerical procedure in [11] is extended following the approach in [21] to accommodate interfaces between inert and reactive media. Detonation propagation in a two-dimensional rate stick is considered first to classify the confinement as strong or weak (following the discussion in [22]) and to establish the convergence and accuracy of numerical solutions for both types of confinement. The numerical method is then used to obtain solutions for the corner-turning and cone problems mentioned above, but it also worth noting that the numerical approach developed here is quite general and can be applied to a wide range of nonlinear, hyperbolic differential equations governing multi-material, reactive systems in both two-dimensional and axisymmetric configurations. A further development of a three-dimensional, parallel capability is in progress.

The remaining sections of the paper are organized as follows. In Section 2 we describe the governing equations of the mathematical model, including the equation of state for the multi-material flow and the multistage reaction rate of the IG model. The numerical method used to compute solutions of the governing equations is discussed in Section 3. Here we provide a brief description of the overlapping grid framework, including our scheme of block-structured AMR as well as descriptions of the fractional step scheme and the energy correction. Our treatment of the equation of state, which for the multi-material flow is defined implicitly, is also discussed. Numerical results are presented in Section 4, and concluding remarks are made in Section 5.

\section{Governing Equations}

We consider a mixture of two inviscid, compressible materials, one the reactive explosive and the other the inert confiner. The equations are presented for two-dimensional flow. The density of the mixture is denoted by $\rho$, the components of velocity by $\left(u_{1}, u_{2}\right)$, the pressure by $p$, and the total energy by $E$, with each 
quantity depending on position $\left(x_{1}, x_{2}\right)$ and time $t$. These quantities satisfy the usual balance equations

$$
\frac{\partial}{\partial t}\left[\begin{array}{c}
\rho \\
\rho u_{1} \\
\rho u_{2} \\
E
\end{array}\right]+\frac{\partial}{\partial x_{1}}\left[\begin{array}{c}
\rho u_{1} \\
\rho u_{1}^{2}+p \\
\rho u_{1} u_{2} \\
u_{1}(E+p)
\end{array}\right]+\frac{\partial}{\partial x_{2}}\left[\begin{array}{c}
\rho u_{2} \\
\rho u_{1} u_{2} \\
\rho u_{2}^{2}+p \\
u_{2}(E+p)
\end{array}\right]=0,
$$

representing conservation of mass, momentum and energy for the mixture. The composition of the mixture is determined by the mass fraction $\phi_{r}$ of the reactive material and the mass fraction $\phi_{i}=1-\phi_{r}$ of the inert material. We assume no mass transfer between the inert and reactive materials so that $\phi_{r}$ satisfies

$$
\frac{\partial \phi_{r}}{\partial t}+u_{1} \frac{\partial \phi_{r}}{\partial x_{1}}+u_{2} \frac{\partial \phi_{r}}{\partial x_{2}}=0
$$

(A similar equation holds for $\phi_{i}$ ). In addition, we let $\lambda$ denote the progress of reaction from the unreacted solid explosive, when $\lambda=0$, to the gaseous products of reaction, when $\lambda=1$. The reaction rate is taken to be $\mathcal{R}$ (discussed in Section 2.2) so that $\lambda$ satisfies the equation

$$
\frac{\partial \lambda}{\partial t}+u_{1} \frac{\partial \lambda}{\partial x_{1}}+u_{2} \frac{\partial \lambda}{\partial x_{2}}=\mathcal{R}
$$

The advection equations in (2) and (3) may be combined with the balance equations in (1) to give the system of governing equations

$$
\frac{\partial}{\partial t} \mathbf{u}+\frac{\partial}{\partial x_{1}} \mathbf{f}_{1}(\mathbf{u})+\frac{\partial}{\partial x_{2}} \mathbf{f}_{2}(\mathbf{u})=\mathbf{R}
$$

where

$$
\mathbf{u}=\left[\begin{array}{c}
\rho \\
\rho u_{1} \\
\rho u_{2} \\
E \\
\rho \phi_{r} \\
\rho \lambda
\end{array}\right], \quad \mathbf{f}_{1}(\mathbf{u})=\left[\begin{array}{c}
\rho u_{1} \\
\rho u_{1}^{2}+p \\
\rho u_{1} u_{2} \\
u_{1}(E+p) \\
\rho u_{1} \phi_{r} \\
\rho u_{1} \lambda
\end{array}\right], \quad \mathbf{f}_{2}(\mathbf{u})=\left[\begin{array}{c}
\rho u_{2} \\
\rho u_{1} u_{2} \\
\rho u_{2}{ }^{2}+p \\
u_{2}(E+p) \\
\rho u_{2} \phi_{r} \\
\rho u_{2} \lambda
\end{array}\right], \quad \mathbf{R}=\left[\begin{array}{c}
0 \\
0 \\
0 \\
0 \\
0 \\
\rho \mathcal{R}
\end{array}\right]
$$

The total energy for the mixture is given by

$$
E=\rho e+\frac{1}{2} \rho\left(u_{1}^{2}+u_{2}^{2}\right) .
$$

where $e=e\left(\rho, p, \phi_{r}, \lambda\right)$ is the specific internal energy, prescribed by an equation of state for the mixture. This equation of state is discussed in detail in Section 2.1 below.

Several problems considered later in Section 4 are axisymmetric. For these problems a geometric source term is included in (4). Assuming $x_{2}=0$ is the axis of symmetry, the source term in (4) becomes

$$
\mathbf{R}=\left[\begin{array}{c}
0 \\
0 \\
0 \\
0 \\
0 \\
\rho \mathcal{R}
\end{array}\right]-\frac{u_{2}}{x_{2}}\left[\begin{array}{c}
\rho \\
\rho u_{1} \\
\rho u_{2} \\
E+p \\
\rho \phi_{r} \\
\rho \lambda
\end{array}\right]
$$

This geometric term in (6) is not stiff and presents no essential difficulty for a numerical treatment, although some special care is needed to handle the removable singularity at $x_{2}=0$.

The variables in (4) and (5), and all subsequent equations, are assumed to be dimensionless. They are obtained by scaling the dimensional variables with corresponding dimensional reference quantities, denoted by $\rho_{\text {ref }}, u_{\text {ref }}, p_{\text {ref }}$, etc.. Choices for these reference quantities are given later in the context of the specific problems studied. In addition, the independent variables in the equations are made dimensionless by scaling them with reference scales for time and length given by $t_{\text {ref }}$ and $x_{\text {ref }}=t_{\text {ref }} u_{\text {ref }}$, respectively. 
In general, we will consider problems in which a sharp interface (a contact discontinuity) separates the inert material where $\phi_{r}=0$ from the reactive material where $\phi_{r}=1$. Throughout this paper, a subscript $i$ will be used to denote quantities belonging to the inert material. For the reactive material, a subscript $s$ will be used to denote the solid explosive while a subscript $g$ will be used to denote the gaseous products.

\section{$2.1 \quad$ Equation of State}

An equation of state is needed to specify the internal energy $e\left(\rho, p, \phi_{r}, \lambda\right)$ for the mixture in (5) in terms of the density and pressure of the mixture, and the mass fractions of the reactive material and the gaseous products of reaction. For the problems considered in this paper, a mixture of solid explosive and gaseos products occurs in the reaction zone where $0<\lambda<1$, and in the smeared interface between the reactive component and the inert where $0<\phi_{r}<1$. (The latter occurs as a result of the numerical capturing method and requires special treatment as is discussed in Section 3.3.) Following the modeling approach used by Tarver and co-workers in $[1,2,3]$ for the ignition-and-growth (IG) model, we construct an equation of state for the mixture based on a mass-weighted average of constituent equations of state together with assumed closure rules. For the constituent materials, we assume mechanical and thermal equations of state of Jones-Wilkins-Lee (JWL) form, a special case of the Mie-Grüneisen equation of state [23]. These have the form

$$
\left.\begin{array}{rl}
e_{k} & =\frac{p_{k} v_{k}}{\omega_{k}}-\mathcal{F}_{k}\left(v_{k}\right)+\mathcal{F}_{k}\left(v_{0, k}\right)+Q_{k}, \\
p_{k} & =\frac{\omega_{k}}{v_{k}}\left(C_{v, k} T_{k}+\mathcal{Z}_{k}\left(v_{k}\right)-\mathcal{Z}_{k}\left(v_{0, k}\right)\right),
\end{array}\right\}, \quad k=i, s \text { or } g,
$$

where $v_{k}, p_{k}, e_{k}$ and $T_{k}$ are the specific volume, pressure, specific energy and temperature, respectively, for material $k$, and $v_{0, k}$ is a reference specific volume. Material $k$ is specified by its Grüneisen constant $\omega_{k}$ and specific heat at constant volume $C_{v, k}$, and by the stiffening functions, $\mathcal{F}_{k}$ and $\mathcal{Z}_{k}$, which are given by

and

$$
\mathcal{F}_{k}\left(v_{k}\right)=A_{k}\left(\frac{v_{k}}{\omega_{k}}-\frac{1}{R_{1, k}}\right) \exp \left(-R_{1, k} v_{k}\right)+B_{k}\left(\frac{v_{k}}{\omega_{k}}-\frac{1}{R_{2, k}}\right) \exp \left(-R_{2, k} v_{k}\right)
$$

$$
\mathcal{Z}_{k}\left(v_{k}\right)=A_{k}\left(\frac{v_{k}}{\omega_{k}}\right) \exp \left(-R_{1, k} v_{k}\right)+B_{k}\left(\frac{v_{k}}{\omega_{k}}\right) \exp \left(-R_{2, k} v_{k}\right)
$$

where $A_{k}, B_{k}, R_{1, k}, R_{2, k}$ are constants. These functions are fit to experimental data and the constants in (8) and (9) are available for a large number of materials at various conditions [24]. Note that the JWL forms in (7) also include the ideal gas case when $\mathcal{F}_{k}=\mathcal{Z}_{k}=0$. Finally $Q_{k}$ is the reference internal energy for each material. We set $Q_{g}=0$ so that $Q_{s}>0$ measures the heat released as solid explosive converts to gaseous products. The reference internal energy $Q_{i}$ may be chosen for convenience for each simulation because no mechanism to release this energy exists (i.e. there is no source term in the equation for $\phi_{r}$ ).

The specific volume $v=1 / \rho$ for the mixture is related to the volumes $v_{k}, k=i, s$ and $g$, of the constituents by the weighted average

$$
v=\phi_{r}\left[\lambda v_{g}+(1-\lambda) v_{s}\right]+\left(1-\phi_{r}\right) v_{i} .
$$

Similarly, the internal energy for the mixture is related to those of the constituents by

$$
e=\phi_{r}\left[\lambda e_{g}+(1-\lambda) e_{s}\right]+\left(1-\phi_{r}\right) e_{i} .
$$

Following the work in $[1,2,3]$, we assume pressure and temperature equilibrium so that $p=p_{i}=p_{s}=p_{g}$ and $T_{i}=T_{s}=T_{g}$. These closure conditions provide the final equations needed to specify (implicitly) an equation of state for the mixture. These implicit equations are evaluated numerically and the details of this evaluation are provided in Section 3.4.

\subsection{Reaction Rate}

For the purposes of this paper, we consider two reaction rate laws. The first is a relatively simple pressuredependent rate law given by

$$
\mathcal{R}=\sigma(1-\lambda)^{\nu} p^{n}
$$


where $\sigma, \nu$ and $n$ are positive constants. Reaction rates such as the simple choice above which depend on pressure explicitly are often preferred for mathematical models of detonations in solid explosives, in part because the experimental apparatus can measure pressure whereas other physical quantities such as temperature are difficult or impossible to measure. Such rate laws are then be fit to experimental data for a given material. Furthermore, the stability properties and the reaction-zone structure of steady planar detonations for the rate law in (12) are known and understood [23, 25].

The second rate law considered here is the one used in the IG model $[1,2,3]$. It may be regarded as an extension of the one in (12), and provides a multi-stage, phenomenological, macroscopic description of the microscopic behavior of condensed-phase explosives. It is given by

$$
\mathcal{R}=\mathcal{R}_{I}+\mathcal{R}_{G_{1}}+\mathcal{R}_{G_{2}}
$$

where

$$
\begin{aligned}
& \mathcal{R}_{I}= \begin{cases}0 & \text { if } \rho<1+a, \\
I(1-\lambda)^{b}(\rho-1-a)^{x} & \text { if } \rho \geq 1+a \text { and } \lambda<\lambda_{\mathrm{ig}, \max },\end{cases} \\
& \mathcal{R}_{G_{1}}= \begin{cases}G_{1}(1-\lambda)^{c} \lambda^{d} p^{y} & \text { if } 0<\lambda \leq \lambda_{G_{1}, \text { max }}, \\
0 & \text { if } \lambda>\lambda_{G_{1}, \max },\end{cases} \\
& \mathcal{R}_{G_{2}}= \begin{cases}0 & \text { if } \lambda<\lambda_{G_{2}, \text { min }}, \\
G_{2}(1-\lambda)^{e} \lambda^{g} p^{z} & \text { if } \lambda \geq \lambda_{G_{2}, \text { min }} .\end{cases}
\end{aligned}
$$

Here, $\mathcal{R}_{I}$ is an ignition term and $\mathcal{R}_{G_{1}}$ and $\mathcal{R}_{G_{2}}$ are growth terms. There are a large number of parameters that appear, namely $I, b, a, x, G_{1}, c, d, y, G_{2}, e, g$, and $z$, and these are chosen based on the particular explosive to be modeled and the particular regime of detonation, either detonation initiation or propagation [3, 10]. The ignition term is introduced to model hot-spot formation due to shock compression. Of particular note is the compression threshold, $\rho_{\text {ign }}=1+a$. Ignition is assumed to occur when the mixture density (made dimensionless with the undisturbed density of the solid explosive) rises above $\rho_{\mathrm{ign}}$. Once ignition occurs, $\lambda$ rises above zero and the growth terms turn on. The first growth term, given by $\mathcal{R}_{G_{1}}$, models the spread of hot spots through the bulk of the explosive and is active when $0<\lambda \leq \lambda_{G_{1}, \max }$, whereas the second growth term, given by $\mathcal{R}_{G_{2}}$, models the coalescence of the hot spots and is active when $\lambda \geq \lambda_{G_{2} \text {,min }}$. The parameters for each stage of the rate law are fit to experimental data and use assumed knowledge about the structure of the specific explosive being modeled.

\section{Numerical Method}

The numerical method used to solve the governing equations in (4) with a mixture EOS and chosen reaction rate is a high-resolution Godunov method. The equations are discretized on an overlapping grid consisting of a set of curvilinear component grids, as discussed in Section 3.1, in order to handle the complex flow geometry. Adaptive mesh refinement (AMR) is incorporated to locally increase the grid resolution near contacts, shocks and detonations. A Strang-type fractional-step scheme is used to advance the equations in time. One step handles the nonlinear convection portion of the equations given by (4) with the righthand-side set to zero, while the other step considers the reaction rate alone given by either (12) or (13). Details are given in Section 3.2. In a typical calculation, the structure of the reaction zone where $\phi_{r}=1$ and $0<\lambda<1$ is well-resolved using many cells on the finest grid level. The material interface, on the other hand, where $\phi_{r}$ changes abruptly from 0 to 1 is smeared over a few grid cells on the finest grid level. An energy correction is included in the method in order to avoid numerical oscillations near the interface that would occur otherwise due to the shock-capture scheme. This approach follows the work in [21] and is discussed briefly in Section 3.3. We close the discussion of the numerical method in Section 3.4 by describing our numerical treatment of the mixture equation of state. 


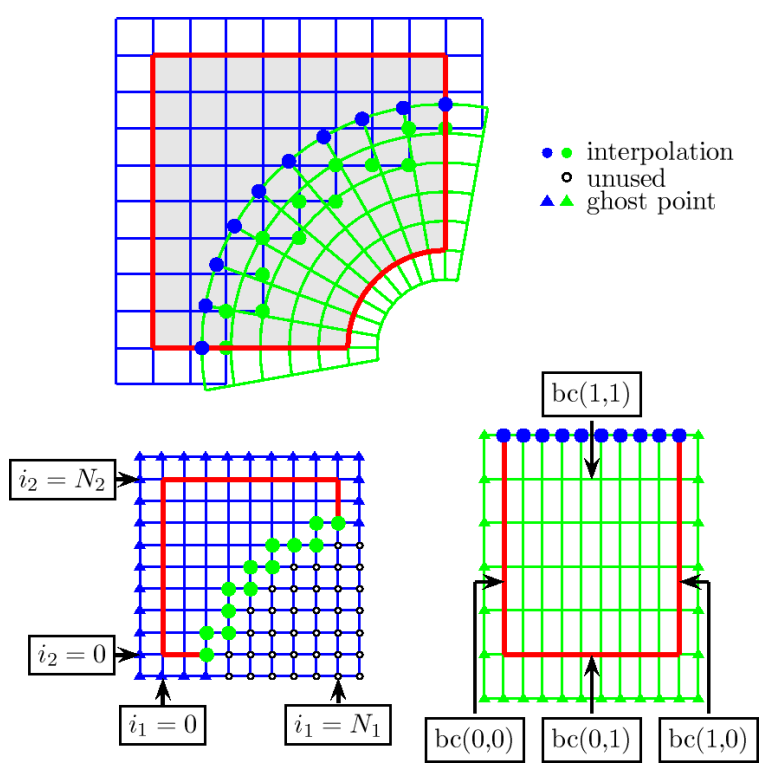

Figure 1: The top view shows an overlapping grid consisting of two structured curvilinear component grids. The bottom views show the component grids in the unit square parameter space. Grid points are classified as discretization points, interpolation points (green or blue dots), or unused points (open disks). Ghost points (green or blue triangles) are used to apply boundary conditions.

\subsection{Overlapping grid framework}

In order to perform computations on complicated flow geometries we assume that the domain $\Omega$ is discretized by an overlapping grid $\mathcal{G}$. The overlapping grid consists of a set of component grids $\left\{\mathcal{G}_{j}\right\}, j=1, \ldots, \mathcal{N}_{g}$, that cover $\Omega$ and overlap where they meet. Typically the bulk of the domain is covered by Cartesian grids while smooth boundary-fitted grids are used to represent the boundary of the domain. Each component grid covers a sub-domain $\Omega_{j}$ of $\Omega$ in physical space and is defined by a smooth mapping from the physical space $\left(x_{1}, x_{2}\right)$ in two dimensions to the unit square $\left(r_{1}, r_{2}\right)$ in computational space. The mapping is applied to the governing equations, and the mapped equations are discretized assuming a general curvilinear grid. For the case of Cartesian grids, the discretization is greatly simplified. We implement this simplification as a special case in our computational kernels for numerical efficiency.

A simple example of an overlapping grid is shown in figure 1. In this example, the overlapping grid $\mathcal{G}$ consists of a background Cartesian grid and a boundary-fitted annular grid. The annular grid cuts a hole in the background grid rendering some grid points unused. These unused points are tagged and no computation is performed there. The remaining grid points are either interpolation points or discretization points. Interpolation points are those where the numerical solution is communicated between grids at the overlap. Discretization points are those where the discretization of the governing equations or boundary conditions are applied. The grid also employs ghost points to facilitate the numerical approximation of boundary conditions. The discretization described later requires two layers of ghost points to support the width of the 5-point second-order Godunov stencil.

The interpolation at the overlap between grids may be performed using either primitive variables $\mathbf{w}=$ $\left[\rho, u_{1}, u_{2}, p, \phi_{r}, \lambda\right]^{T}$ or conservative variables $\mathbf{u}=\left[\rho, \rho u_{1}, \rho u_{2}, E, \rho \phi_{r}, \rho \lambda\right]^{T}$. In this paper we adopt the interpolation method described in [21] which uses bi-linear interpolation in terms of primitive variables. These primitive quantities are used, rather than conservative quantities as in [12], in order to avoid numerical oscillations that would develop otherwise near an intersection of the material interface and the grid overlap. Concerns relating to the lack of discrete conservation as a result of these interpolation schemes are addressed at length in [21] through a number of test problems and convergence studies. One can also see [26, 27] for a more in depth discussion of the the effects of conservation at grid overlap. 


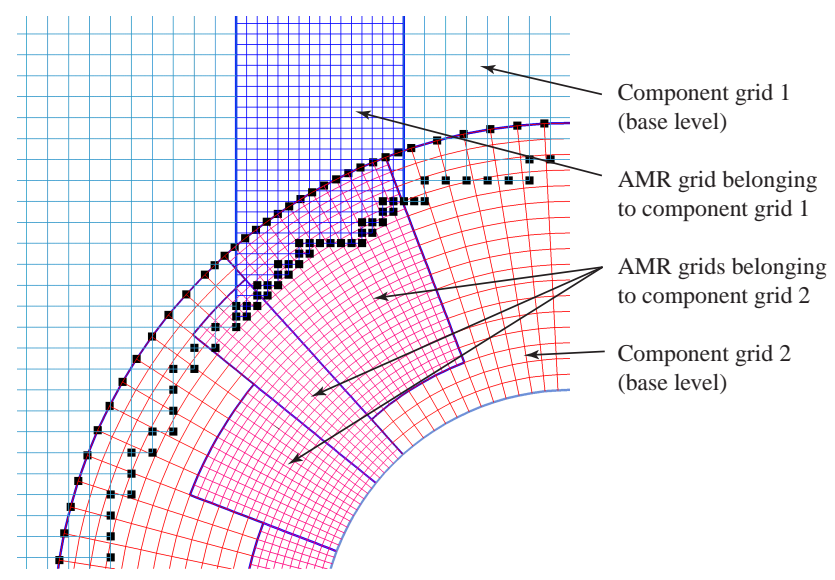

Figure 2: Overlapping grids and AMR; a view of the overlap region showing the interpolation between refinement grids from different base grids. The black squares indicate interpolation points.

Adaptive mesh refinement (AMR) is used in regions of the flow where the solution changes rapidly, such as near shocks, detonations, and material interfaces, while maintaining coarser grids where the solution is smooth. This allows us to perform calculations at a much finer resolution than would be possible had the entire domain been covered by the finest mesh. Within the overlapping grid framework each AMR grid is defined as a restriction of the smooth mapping from physical space to computational space belonging to the coarser parent grid. In this manner AMR grids retain the smooth description of the boundary. These refinement grids are treated identically to coarser grids within most computational kernels and so implementation of the AMR algorithm is greatly simplified. We employ a block-structured AMR approach following that described originally in [28] and use modifications for overlapping grids as presented in [12]. An estimate of the error is used to tag grid points for refinement. Following [12], we use

$$
\mathbf{e}_{\mathbf{i}}=\sum_{k=1}^{m} e_{\mathbf{i}}^{(k)}+\frac{c_{3}}{s_{k}} \tau_{\mathbf{i}}
$$

where $m$ is the number of components, $\tau_{\mathbf{i}}$ is an estimate of the truncation error from the integration of the reaction rate law at cell $\mathbf{i}$, and

$$
e_{\mathbf{i}}^{(k)}=\frac{1}{2} \sum_{\alpha=1}^{2}\left(\frac{c_{1}}{s_{k}}\left|\Delta_{0 \alpha} U_{\mathbf{i}}^{(k)}\right|+\frac{c_{2}}{s_{k}}\left|\Delta_{+\alpha} \Delta_{-\alpha} U_{\mathbf{i}}^{(k)}\right|\right)
$$

is an estimate of the error in the $k^{\text {th }}$ component of $U_{\mathbf{i}}$, the numerical solution at cell $\mathbf{i}$. In (15), $s_{k}$ is a scale factor for $U_{\mathbf{i}}^{(k)}, c_{1}, c_{2}$, and $c_{3}$ are constants (weights), and $\Delta_{0 \alpha}, \Delta_{+\alpha}$ and $\Delta_{-\alpha}$ are the un-divided central, forward and backward difference operators, respectively, in the $\alpha$-direction in index space. The error estimate is computed for each component grid, and then smoothed and interpolated where the grids overlap. Refinement grid patches are created to cover all grids points where the smoothed error estimate is greater than a chosen tolerance. For example, figure 2 shows the refinement grid structure near an overlap between grids. For the problems considered in this work, the material interface and all detonation structures lie on the finest refinement grid level.

\subsection{Fractional-step scheme}

The computations presented in this paper are performed using a fractional-step scheme which computes a full update from time $t_{n}$ to time $t_{n+1}=t_{n}+\Delta t$ through application of the reaction and hydrodynamic operators separately. Let $U_{\mathbf{i}}^{n}$ be the numerical solution at grid cell $\mathbf{i}$ on a particular component grid and at a time level $t_{n}$. The numerical solution at time level $t_{n+1}$ is given by

$$
U_{\mathbf{i}}^{n+1}=\mathcal{S}_{R}(\Delta t / 2) \mathcal{S}_{H}(\Delta t) \mathcal{S}_{R}(\Delta t / 2) U_{\mathbf{i}}^{n},
$$


where $\mathcal{S}_{R}$ and $\mathcal{S}_{H}$ denote numerical approximations of the reaction and hydrodynamic operators, respectively, and $\Delta t$ is a global time step chosen according to a CFL stability constraint.

The two reaction steps in (16) are performed by solving the ordinary differential equations

$$
\frac{\partial}{\partial t} \mathbf{u}=\mathbf{R}
$$

from (4) over a time $\Delta t / 2$. These equations reduce to the scalar ODE

$$
\frac{\partial}{\partial t} \lambda=\mathcal{R}, \quad \text { with }\left(\rho, \rho u_{1}, \rho u_{2}, E, \rho \phi_{r}\right) \text { held fixed }
$$

which is solved numerically using an adaptive Runge-Kutta error-control scheme as described in [12, 29]. This second-order scheme allows sub-CFL time steps at grid cells where the reaction is active, and delivers an estimate for the truncation error $\tau_{\mathrm{i}}$ which is used in (14). We use this estimate to tag cells for refinement which, in turn, results in a reduced CFL time step, $\Delta t$, so that typically at most 2 or 3 sub-CFL steps are taken for any grid cell.

The hydrodynamic step in (16) involves the nonlinear convective portion of the governing equations, namely,

$$
\frac{\partial}{\partial t} \mathbf{u}+\frac{\partial}{\partial x_{1}} \mathbf{f}_{1}(\mathbf{u})+\frac{\partial}{\partial x_{2}} \mathbf{f}_{2}(\mathbf{u})=0 .
$$

Within the framework of overlapping grids, each component grid (including base-level grids and any refined grids) is defined by a mapping from physical space $\left(x_{1}, x_{2}\right)$ to the unit square in computational space $\left(r_{1}, r_{2}\right)$. In computational space, equation (19) becomes

$$
\frac{\partial}{\partial t} \mathbf{u}+\frac{1}{J} \frac{\partial}{\partial r_{1}} \mathbf{F}_{1}(\mathbf{u})+\frac{1}{J} \frac{\partial}{\partial r_{2}} \mathbf{F}_{2}(\mathbf{u})=0
$$

where

$$
\mathbf{F}_{1}(\mathbf{u})=\frac{\partial x_{2}}{\partial r_{2}} \mathbf{f}_{1}-\frac{\partial x_{1}}{\partial r_{2}} \mathbf{f}_{2}, \quad \mathbf{F}_{2}(\mathbf{u})=-\frac{\partial x_{2}}{\partial r_{1}} \mathbf{f}_{1}+\frac{\partial x_{1}}{\partial r_{1}} \mathbf{f}_{2}
$$

and

$$
J=\left|\frac{\partial\left(x_{1}, x_{2}\right)}{\partial\left(r_{1}, r_{2}\right)}\right| .
$$

The metrics of the mapping, $\frac{\partial x_{1}}{\partial r_{2}}, \frac{\partial x_{2}}{\partial r_{2}}$, etc., and the Jacobian, $J$, are known for each component grid. The discretization of (20) is performed using a uniform grid $\left(r_{1, i}, r_{2, j}\right)$ with grid spacing $\left(\Delta r_{1}, \Delta r_{2}\right)$. Following the approach taken in [21], a quasi-conservative scheme is used in order to suppress numerical oscillations near material interfaces. This scheme is written in the form

$$
U_{i, j}^{*}=\hat{U}_{i, j}^{*}+\Delta G_{i, j}^{*}
$$

where

$$
\hat{U}_{i, j}^{*}=U_{i, j}^{\prime}-\frac{\Delta t}{J \Delta r_{1}}\left(F_{1, i+1 / 2, j}-F_{1, i-1 / 2, j}\right)-\frac{\Delta t}{J \Delta r_{2}}\left(F_{2, i, j+1 / 2}-F_{2, i, j-1 / 2}\right) .
$$

Here, $U_{i, j}^{\prime}=\mathcal{S}_{R}(\Delta t / 2) U_{\mathrm{i}}^{n}$ is the numerical solution, an approximation to the cell average at $\mathbf{i}=(i, j)$, after the first reaction step, $F_{1, i \pm 1 / 2, j}$ and $F_{2, i, j \pm 1 / 2}$ are numerical fluxes in the $r_{1}$ and $r_{2}$ directions, respectively, and $U_{i, j}^{*}=\mathcal{S}_{H}(\Delta t) U_{i, j}^{\prime}$ is the result of the hydrodynamic step. The numerical fluxes are approximations of the mapped fluxes in (21), and these are computed using a second-order, slope-corrected Godunov scheme with an approximate Roe Riemann solver as described in [21]. A small, non-conservative correction given by

$$
\Delta G_{i, j}^{*}=\left[0,0,0, \Delta E_{i, j}^{*}, 0,0\right]^{T}
$$

is added to the cell average, $\hat{U}_{i, j}^{*}$, from the conservative scheme in (23). This term involves an energy correction, $\Delta E_{i, j}^{*}$, which turns on near a material interface and is designed to suppress numerical oscillations there as we discuss in the next section. 


\subsection{Energy correction}

The motivation for the correction, $\Delta G_{i, j}^{*}$, in (24) stems from the observation that an application of a standard shock-capturing scheme, such as Godunov's method, to the conservative system in (19) produces numerical oscillations near the smeared material interface. The situation is illustrated in figure 3 where Godunov's method is applied to a one-dimensional version of (19) with initial conditions $\left(\rho, u, p, \phi_{r}, \lambda\right)=$ $(0.138,0.5,1.0,1.0,1.0)$ for $x<0.4$ and $(1.0,0.5,1.0,0.0,1.0)$ for $x \geq 0.4$. The interface, initially at $x=0.4$, separates material $r$ where $\phi_{r}=1.0$ on the left from material $i$ where $\phi_{r}=0.0$ on the right. For simplicity of illustration, both materials are taken to be ideal gases with $\gamma_{r}=\gamma_{g}=1.67$ and $C_{v, r}=C_{v, g}=3.11$ (since $\lambda=1$ ) and $\gamma_{i}=1.4$ and $C_{v, i}=0.72$. The material interface propagates to the right with speed equal to 0.5 in the exact solution for this uniform pressure-velocity (UPV) flow. This solution is shown at $t=0.1$ by the black curves in figure 3 , while the red dots show the corresponding numerical solution. We observe that the numerical values for $\rho$ and $\phi_{r}$ are in reasonably good agreement with the exact solution, but that the values for $u$ and $p$ are not. The smeared interface generates significant numerical errors in these latter two quantities that propagate away from the interface along the forward and backward characteristic waves, $u \pm c$, where $c$ is the sound speed for the appropriate material state.
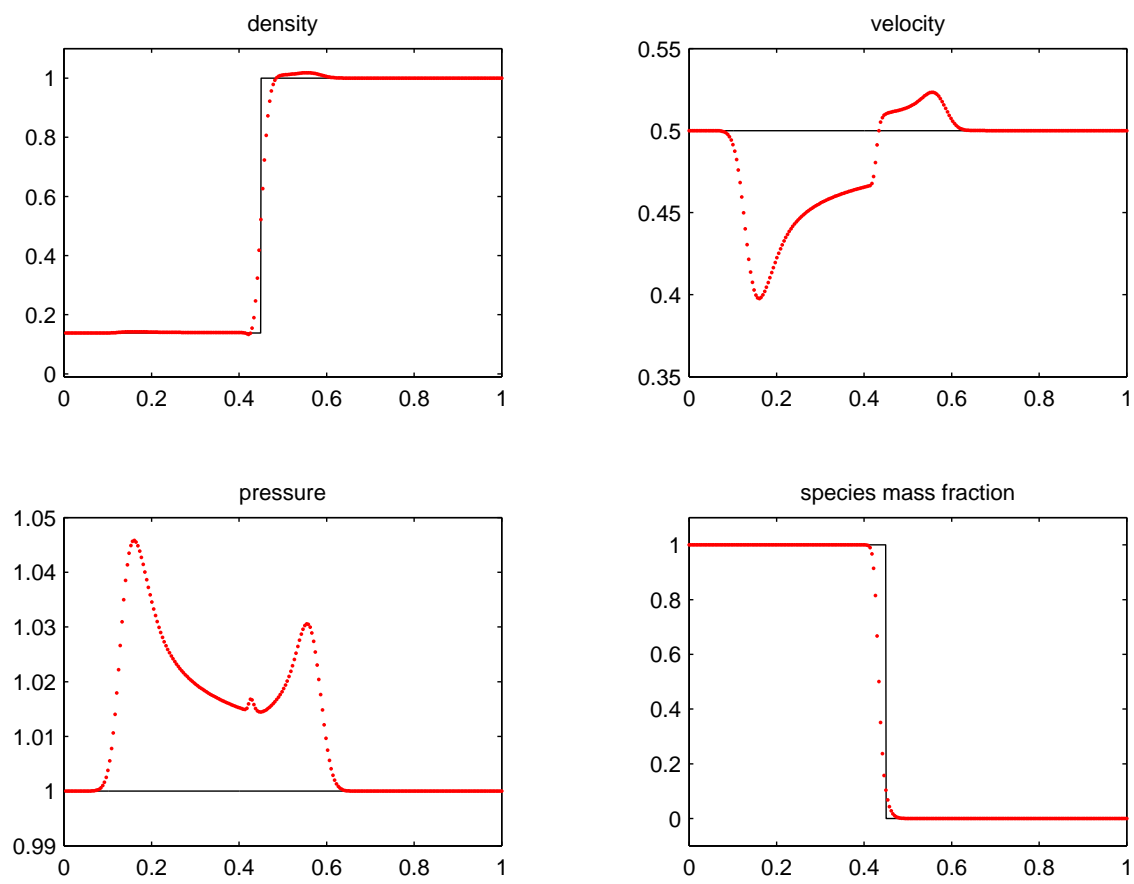

Figure 3: Numerical solution at $t=0.1$ using Godunov's method with $\Delta x=.004$ and CFL number equal to 0.8 . The exact solution is shown by the black curves and the numerical solution is shown by the red dots.

Following the work in [21], an energy correction is used to eliminate unphysical behavior associated with the use of a shock-capturing scheme for UPV flow. We consider the two step process in (22) and (23) with the intermediate state $\hat{U}_{i, j}^{*}$ being the result of the conservative slope-corrected Godunov scheme in (23). As such $\hat{U}_{i, j}^{*}$ is potentially contaminated by the type of error illustrated in figure 3 , and an energy correction is added to suppress this numerical error. Elimination of the error is achieved by performing an auxiliary calculation for a suitable UPV flow to determine the size of the error. Let

$$
\tilde{U}_{i, j}^{*}=U_{i, j}^{\prime}-\frac{\Delta t}{J \Delta r_{1}}\left(\tilde{F}_{1, i+1 / 2, j}-\tilde{F}_{1, i-1 / 2, j}\right)-\frac{\Delta t}{J \Delta r_{2}}\left(\tilde{F}_{2, i, j+1 / 2}-\tilde{F}_{2, i, j-1 / 2}\right),
$$

where $\tilde{F}_{1, i \pm 1 / 2, j}$ and $\tilde{F}_{2, i, j \pm 1 / 2}$ are numerical fluxes obtained using left and right states corresponding to a 
UPV flow determined by the velocity and pressure given by $U_{i, j}^{\prime}$. For example, $\tilde{F}_{1, i+1 / 2, j}$ is computed by solving a Riemann problem with left and right states given by $\tilde{U}_{L}$ and $\tilde{U}_{R}$, respectively, corresponding to the states $U_{L}$ and $U_{R}$ used in the calculation of $F_{1, i+1 / 2, j}$ in (23), but with the velocity and pressure in both states replaced by $\left(u_{1, j}^{\prime}, u_{2, j}^{\prime}\right)$ and $p_{i, j}^{\prime}$, respectively. Hence, the solution of the Riemann problem used to determine $\tilde{F}_{1, i+1 / 2, j}$ consists only of a contact discontinuity, and so its calculation and the corresponding calculation for $\tilde{U}_{i, j}^{*}$ in (25) are straightforward.

We now have the necessary information to compute the energy correction in (22). Let $p_{i, j}^{\prime}$ and $\tilde{p}_{i, j}^{*}$ be the pressures given by the states $U_{i, j}^{\prime}$ and $\tilde{U}_{i, j}^{*}$, respectively, and define

$$
\Delta p_{i, j}^{*}=p_{i, j}^{\prime}-\tilde{p}_{i, j}^{*} .
$$

The energy correction is then given by

$$
\Delta E_{i, j}^{*}=\hat{\rho}_{i, j}^{*} e\left(\hat{\rho}_{i, j}^{*}, \hat{p}_{i, j}^{*}+\Delta p_{i, j}^{*}, \hat{\phi}_{r i, j}^{*}, \hat{\lambda}_{i, j}^{*}\right)-\hat{\rho}_{i, j}^{*} \hat{e}_{i, j}^{*},
$$

where $\hat{\rho}_{i, j}^{*}, \hat{p}_{i, j}^{*}, \hat{e}_{i, j}^{*}, \hat{\phi}_{r i, j}^{*}$, and $\hat{\lambda}_{i, j}^{*}$ are the density, pressure, internal energy, species mass fraction, and reaction progress given by the state $\hat{U}_{i, j}^{*}$, respectively, and $e=e\left(\rho, p, \phi_{r}, \lambda\right)$ is determined by the equation of state (as discussed in section 3.4).

As noted in [21], the energy correction is nonzero only in regions of the flow where $\phi_{r}$ varies. Thus, for numerical efficiency the calculation of $\tilde{U}_{i, j}^{*}$ in (25) followed by $\Delta E_{i, j}^{*}$ in (26) is performed only near the material interface. This region is narrow and represents a very small fraction of the total number of grid points, and thus the added computational cost of the energy correction is small. Finally, we make the observation that the quasi-conservative scheme in (22) and (23) with the energy correction in (26) is second-order accurate for smooth flow. Further details of the numerical approach are given in [21].

\subsection{Numerical evaluation of the equation of state}

An evaluation of the equation of state for the mixture is required to obtain the internal energy $e$ as a function of the primitive variables $\left(\rho, p, \phi_{r}, \lambda\right)$ for the calculation of the energy correction in (26). We also require an evaluation of the equation of state for the calculation of the Godunov fluxes in (23). For this calculation we need $p$ and its first partial derivatives as functions of the conservative variables $\left(\rho, \rho e, \rho \phi_{r}, \rho \lambda\right)$, in order to obtain eigenvalues and eigenvectors for the Roe Riemann solver. For either form, the evaluation requires a numerical calculation involving the various formulas given in Section 2.1. In special cases where only one of the species is present, the calculation is explicit. For all other cases, the equation of state is defined implicitly and a method of iteration is required as we describe briefly in this section.

Let us begin with the mixture rules in (10) and (11) and define

$$
\alpha_{i}=\left(1-\phi_{r}\right) \rho, \quad \alpha_{s}=(1-\lambda) \phi_{r} \rho, \quad \alpha_{g}=\lambda \phi_{r} \rho,
$$

so that the mixture rules become

$$
1=\sum_{k} \alpha_{k} v_{k}, \quad \rho e=\sum_{k} \alpha_{k} e_{k}, \quad k=\{i, s, g\} .
$$

Using pressure equilibrium and the mechanical JWL equation of state for $e_{k}$ in (7), we have

$$
\rho e=p \sum_{k} \frac{\alpha_{k} v_{k}}{\omega_{k}}-\sum_{k} \alpha_{k}\left(\mathcal{F}_{k}\left(v_{k}\right)-\mathcal{F}_{k}\left(v_{0, k}\right)-Q_{k}\right),
$$

which ultimately gives $e$ as a function of $\left(\rho, p, \phi_{r}, \lambda\right)$ or $p$ as a function of $\left(\rho, \rho e, \rho \phi_{r}, \rho \lambda\right)$ if the species volumes $v_{i}, v_{s}$ and $v_{g}$ are known. The next task, then, is to determine these volumes and this requires a method of iteration since the volumes appear nonlinearly in the equations.

Temperature equilibrium and the three thermal JWL equations of state in (7) give

$$
\frac{1}{C_{v, i}}\left[\frac{p v_{i}}{\omega_{i}}-\mathcal{Z}_{i}\left(v_{i}\right)+\mathcal{Z}_{i}\left(v_{0, i}\right)\right]=\frac{1}{C_{v, s}}\left[\frac{p v_{s}}{\omega_{s}}-\mathcal{Z}_{s}\left(v_{s}\right)+\mathcal{Z}_{s}\left(v_{0, s}\right)\right]=\frac{1}{C_{v, g}}\left[\frac{p v_{g}}{\omega_{g}}-\mathcal{Z}_{g}\left(v_{g}\right)+\mathcal{Z}_{g}\left(v_{0, g}\right)\right] .
$$


Of these equilibrium equations two are independent. So, if $p$ is known, then we may compute $v_{i}, v_{s}$ and $v_{g}$ from two of the equations in (29) and the mixture rule for $v_{k}$ in (27). This may be done using Newton's method and the resulting species volumes are used in (28) to obtain $e$. If, on the other hand, $\rho e$ is known, then we first use (28) to eliminate $p$ from the temperature equilibrium equations in (29). We then use Newton's method to obtain the species volumes from two of the (modified) temperature equilibrium equations and the mixture rule for $v_{k}$ in (27). The resulting species volumes are used in (28) to obtain $p$. For this latter case, the first derivatives of $p$ with respect to $\left(\rho, \rho e, \rho \phi_{r}, \rho \lambda\right)$ may be found explicitly, if needed, once the species volumes are known.

In practice, we save converged values for $v_{i}, v_{s}$ and $v_{g}$ from the previous Newton iteration at each grid point and check for various special cases in order to simplify the evaluation of the EOS and make the numerical calculations more efficient. As mentioned earlier, if only one species is present, then the EOS can be evaluated explicitly. This situation occurs at grid points away from the reaction zone and the material interface and thus is the case for most points on the grid. Another special case occurs for grid points in the reaction zone, but away from the material interface. For these points, $\phi_{r}=1$ (or is within a tolerance of 1) so that the iteration is used to determine $v_{g}$ and $v_{s}$ alone. A similar situation occurs at grid points in the domain well behind the reaction zone where $\lambda \approx 1$ but near the material interface. Here, the iteration need only determine $v_{g}$ and $v_{i}$. For the general case, the iteration proceeds to determine all three specific volumes but this case occurs for a relatively small number of points on the grid.

\section{Numerical Results}

We now consider a range of problems for detonations propagating under compliant confinement. We begin with detonation propagation in a simple rate stick. Here the detonation is initiated by a high-pressure "booster" charge in a two-dimensional slab of explosive bounded by an inert material. As the detonation propagates down the rate stick the high pressure behind it causes the material interface separating the explosive and the inert to deflect from its initial planar position. This release creates a curved detonation front which propagates at a speed less than the steady, planar, Chapman-Jouget (CJ) value. This problem is considered in part to validate the numerical method described in Section 3 and to establish its accuracy. It also serves as a baseline calculation for classifying weak and strong confinement, following the work in [22], and for studying the near-interface behavior under these confinements for a relatively simple geometry.

The second problem involves detonation diffraction and failure in an expanding geometry. Here a steady detonation propagates in a cylindrical rate stick of a chosen diameter and then abruptly turns a $90^{\circ}$ corner as it enters a rate stick of a larger diameter. The geometry is motivated by experiments performed by Ferm et al and reported in [13]. Of particular interest in the experiments was the appearance of a region of unreacted material, or dead zone, in the post-diffracted state where the weakened lead shock of the detonation separated from the reaction zone. Here we examine this problem using the ignition-and-growth (IG) model, with equation-of-state and reaction-rate parameters fit to the explosive PBX 9502 [6]. Both strong and weak confinements are explored, and in the strong-confinement case the results are compared with the corresponding calculations for a rigid confinement. This work is an extension of our earlier study [11] on detonation diffraction under rigid confinement for both two-dimensional and axisymmetric geometries.

The last problem presented in this section examines detonation propagation in a converging geometry. The configuration is that of a cylindrical rate stick tapered at one end to form a pencil-shaped explosive. A detonation initiated at the "eraser" end of the explosive propagates down the rate stick towards the tapered end. Of interest is the behavior of the detonation as it propagates down the taper, into the region where the diameter is below the failure diameter, for various cone angles. The explosive, PBX 9502, is weakly confined, and the numerical results are compared with recent experimental results by Salyer and Hill discussed in [15].

\subsection{Detonation propagation in a two-dimensional rate stick}

A rate stick is a slab or cylinder of solid explosive surrounded by an inert material. The numerical calculations in this subsection consider a two-dimensional slab as shown in figure 4. The rate stick is ignited at its left end via a high-pressure booster, and once a detonation wave forms it propagates to the right through the slab of 


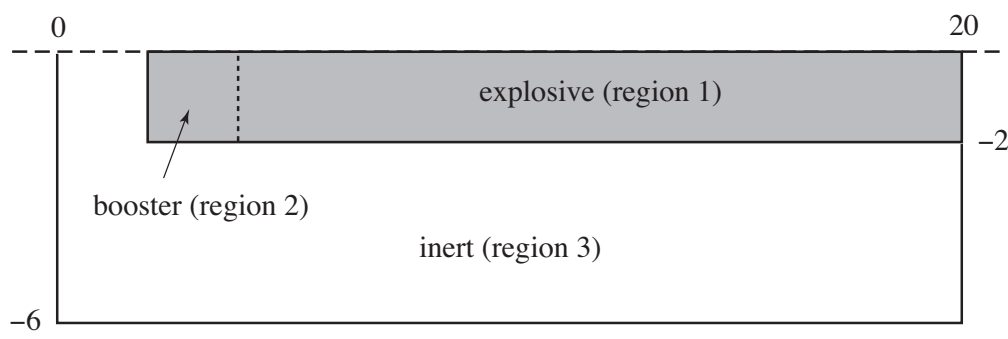

Figure 4: Schematic representation of a rate stick slab geometry. Regions 1 and 2 are explosive material and region 3 is the surrounding inert material. A detonation is initiated in region 1 by a high-pressure booster state in region 2 . The line $x_{2}=0$ is a line of symmetry for the calculation.

reactive material. The surrounding inert material provides a compliant confinement for the explosive, and forced by the high post-detonation pressure, expands outward behind the detonation front. This expansion, in turn, tends to weaken the detonation in the vicinity of the explosive/inert interface, creating a curved detonation front whose unsupported steady velocity is less than the planar CJ value that would occur in a rigidly-confined rate stick.

For this first problem, we model the reactive material, both the explosive and the products of combustion, as well as the inert confiner as ideal polytropic fluids, i.e., we set $\mathcal{F}_{k}=\mathcal{Z}_{k}=0$ for $k=i, s$ and $g$ in (7). For the reactive material, we take

$$
\omega_{s}=\omega_{g}=2, \quad C_{v, s}=C_{v, g}=1, \quad Q_{s}=0.04, \quad Q_{g}=0 .
$$

For the confiner, we make various choices for the equation of state parameters and its ambient density $\rho_{0}$ in order to model weak and strong confinements as discussed below. The reaction rate is taken to be the pressure-dependent law given in (12) with

$$
\sigma=10, \quad \nu=0.5, \quad n=1 .
$$

A small value for $n$ is chosen so that a steady planar CJ detonation is stable and the value for $\nu$ is chosen so that the reaction zone has a finite length $[23,25]$. The explosive in regions 1 and 2 is at rest initially with

$$
\rho_{1}=\rho_{2}=2, \quad \lambda_{1}=\lambda_{2}=0, \quad \phi_{r, 1}=\phi_{r, 2}=1, \quad p_{1}=10^{-6}, \quad p_{2}=0.5,
$$

while the initial state of the inert in region 3 is at rest with

$$
\rho_{3}=\rho_{0}, \quad \lambda_{3}=0, \quad \phi_{r, 3}=0, \quad p_{3}=10^{-6} .
$$

For the exact solution with a sharp interface (a contact discontinuity) separating the reactive material from the inert, the choice for $\lambda$ in the inert at $t=0$ is arbitrary since there is no heat release associated with $\lambda$ when $\phi_{r}=0$. However, for the numerical approximation of the exact solution, the choice for $\lambda$ in the inert at $t=0$ has an effect for $t>0$ in the neighborhood of the smeared interface. For the initial conditions in (33) the choice $\lambda_{3}=0$ is made. We have found this to be a suitable choice, and discuss the effect of other initial values later in Section 4.1.5.

The boundary conditions for the rate-stick geometry shown in figure 4 consist of a symmetry condition at $x_{2}=0$ and an outflow condition on the remaining sides. For later purposes, we note that the speed of the steady, planar CJ detonation for these parameters is well-approximated by the formula for the strong shock limit given by

$$
D_{\mathrm{CJ}}=\sqrt{2\left(\gamma_{s}^{2}-1\right) Q_{s}}=0.8, \quad \gamma_{s}=\omega_{s}+1,
$$

(see [23] and [22]). 

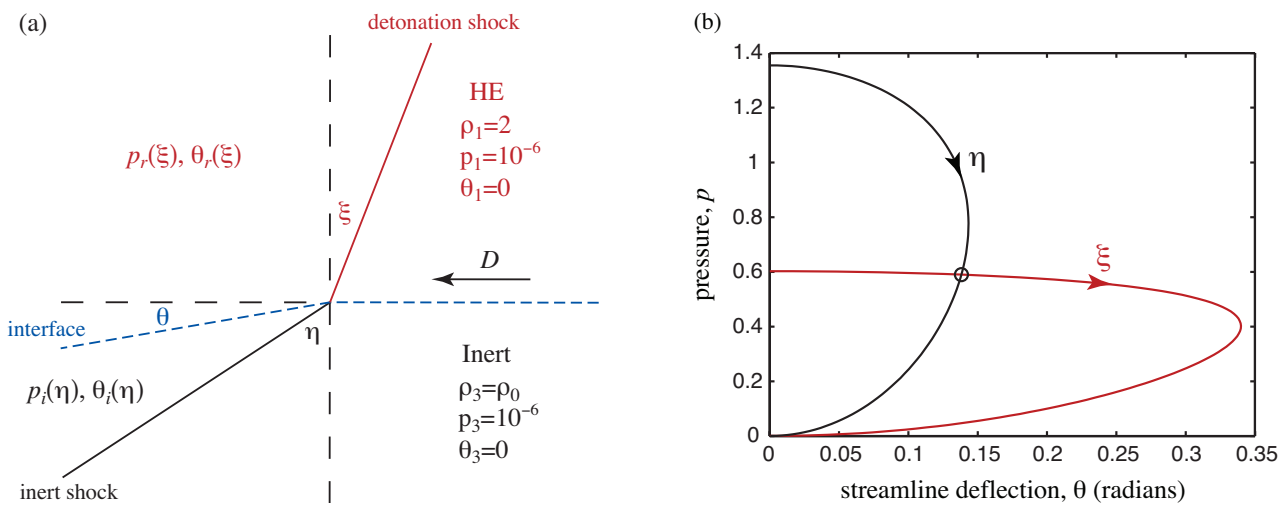

Figure 5: Local behavior near the intersection of the detonation shock and the material interface for a strongly confined detonation: (a) Oblique shock-interface geometry in the frame of the steady structure and (b) shock-polar curves for the HE (red) and inert (black).

\subsubsection{Confinement classification}

In this section we provide a brief description of the local behavior near the intersection of the leading shock of a steady (curved) detonation, and the material interface separating the reactive material from the inert, in order to classify the confinement as strong or weak. This discussion for the ideal fluid case follows that given by Aslam and Bdzil in [22], and a similar approach is used later for calculations involving the mixture JWL equation of state for the IG model. In figure 5(a), for example, we sketch the local behavior near the intersection for a strong confinement case. The sketch is made in the frame of the steady wave so that the flow enters the detonation shock with speed $D$ parallel to the undisturbed interface. The density and pressure ahead of the detonation shock are given by $\rho_{1}$ and $p_{1}$, respectively, with values given in (32), and the flow deflection angle is $\theta_{1}=0$. The pressure and flow deflection immediately behind the detonation shock, denoted by $p_{r}$ and $\theta_{r}$, respectively, are determined by the inclination angle of the shock, $\xi$, and the oblique shock conditions. (The reaction zone behind the detonation shock is not considered for this analysis of post-shock pressure and deflection since its length is of finite extent, see [22].) The detonation in the explosive (HE) creates a downward deflection of the interface behind the detonation shock and an oblique shock in the inert material whose undisturbed state is given by $\rho_{3}=\rho_{0}, p_{3}=10^{-6}$ and $\theta_{3}=0$. The pressure and flow deflection behind the shock in the inert, denoted by $p_{i}$ and $\theta_{i}$, respectively, are determined by the inclination angle, $\eta$, again using the oblique shock conditions. The post-shock states $\left(\theta_{r}(\xi), p_{r}(\xi)\right)$ and $\left(\theta_{i}(\eta), p_{i}(\eta)\right)$ may be plotted in the $\theta-p$ plane for a given value for $D$ as is done in figure $5(\mathrm{~b})$. The value for $D$ may be estimated by $D_{\mathrm{CJ}}=0.8$ given in (34), but we employ the more accurate value $D=0.776$ as determined by the numerical solution of the rate-stick problem with strong confinement (shown in Section 4.1.2). This choice for $D$ along with the given upstream state and the equation of state parameters given in (30) is sufficient to determine the shock-polar curve for the HE. The shock polar for the confiner is obtained for the choice

$$
\rho_{0}=9, \quad \omega_{i}=6, \quad C_{v, i}=1, \quad Q_{i}=0 .
$$

The equilibrium conditions, $\theta_{r}(\xi)=\theta_{i}(\eta)$ and $p_{r}(\xi)=p_{i}(\eta)$, determine the shock inclination angles and the state of the flow on either side of the material interface in the disturbed region immediately behind the shocks. These values are found to be

$$
\xi=0.1439, \quad \eta=0.8503, \quad \theta_{r}=\theta_{i}=0.1382, \quad p_{r}=p_{i}=0.5898
$$

(All angles are given in radians.) The configuration is considered a strong confinement case since the shockpolar curves intersect on the subsonic branch of the HE shock polar, and thus no expansion solution is needed in the HE behind the shock to obtain an equilibrium state. 

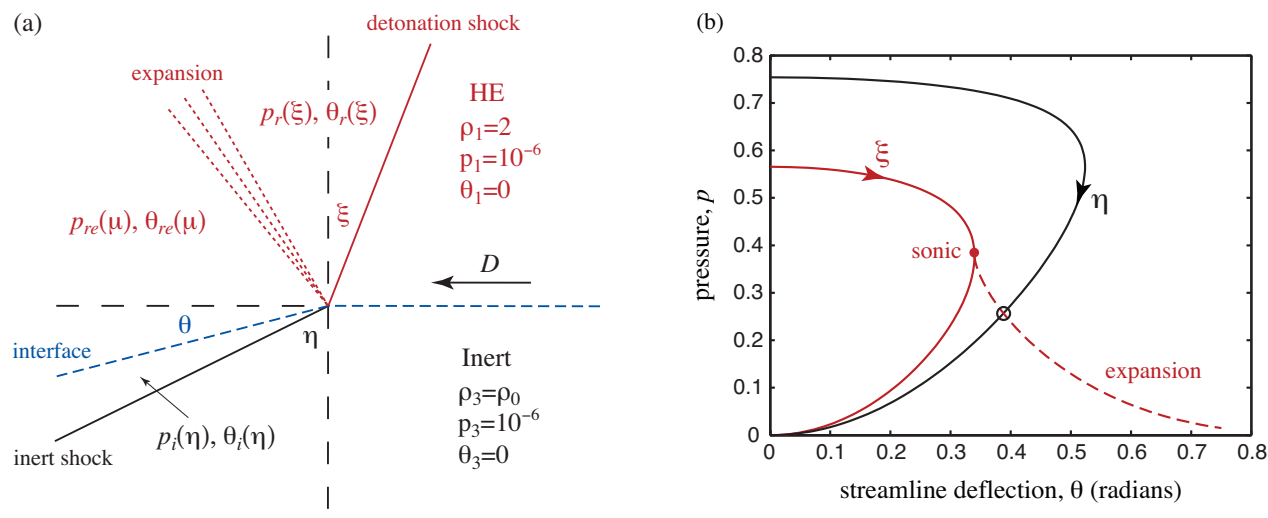

Figure 6: Local behavior near the intersection of the detonation shock and the material interface for a weakly confined detonation: (a) Oblique shock-interface geometry in the frame of the steady structure and (b) shock-polar curves for the HE (red) and inert (black), and Prandtl-Meyer expansion curve for the HE (dashed red curve).

For the weak confinement case, the interface deflection is larger and an expansion solution in the HE is needed to obtain an equilibrium state as illustrated in figure 6(a). For this configuration, the inclination angle for the detonation shock is determined by the condition that the flow behind is sonic. This gives

$$
\xi=\xi_{\mathrm{s}}=0.6155, \quad \theta_{r}=0.3398, \quad p_{r}=0.3770,
$$

using the value $D=0.752$ determined by the numerical solution of the rate stick problem with weak confinement (shown in Section 4.1.3). For an ideal gas equation of state, the sonic value is located at the nose of the shock-polar curve as shown in figure 6(b). The shock polar for the inert for this weak confinement case is obtained for the choice

$$
\rho_{0}=2, \quad \omega_{i}=1, \quad C_{v, i}=1, \quad Q_{i}=0 .
$$

Since the shock-polar curves for the HE and for the inert do not intersect, an expansion solution is needed to obtain an equilibrium state on either side of the material interface. This Prandtl-Meyer expansion solution begins at the sonic point on the shock-polar curve of the HE where the Mach angle is 1, and is parameterized by $\mu$, the Mach angle in the post-expansion state denoted by $\theta_{r e}$ and $p_{r e}$. The locus of these states, $\left(\theta_{r e}(\mu), p_{\text {re }}(\mu)\right)$, may be plotted in the $\theta$ - $p$ plane and the values for $\mu$ and $\eta$ are now obtained from the equilibrium conditions, $\theta_{r e}(\mu)=\theta_{i}(\eta)$ and $p_{r e}(\mu)=p_{i}(\eta)$, which gives

$$
\mu=0.9182, \quad \eta=0.9478, \quad \theta_{r e}=\theta_{i}=0.3879, \quad p_{r e}=p_{i}=0.2567 .
$$

The parameters for the strong and weak confinements listed in (35) and (36), respectively, provide good test cases for the numerical solution of the rate-stick problem, and the local analysis is used later to choose parameters for the JWL equation of state for the inert to obtain strong and weak confinements for the simulations using the IG model.

\subsubsection{Strong confinement}

The case of strong confinement is simulated using an inert material with an ambient density and equation of state parameters given in (35). Figure 7 shows shaded contours of density and pressure of the solution at times $t=5,10,15$ and 20 . The mesh spacing on the base grid for this calculation is $\Delta x_{1}=\Delta x_{2}=0.0625$, and two refinement grid levels are used above the base level to locally increase the grid resolution. A refinement ratio of 4 is used in both directions for this calculation (and for all other calculations in this paper) so that on the finest level the grid spacing is 0.0039 . This level of refinement implies that the width of the reaction zone is spanned by approximately 75 grid cells on the finest grid level. The well-resolved solution shows that 

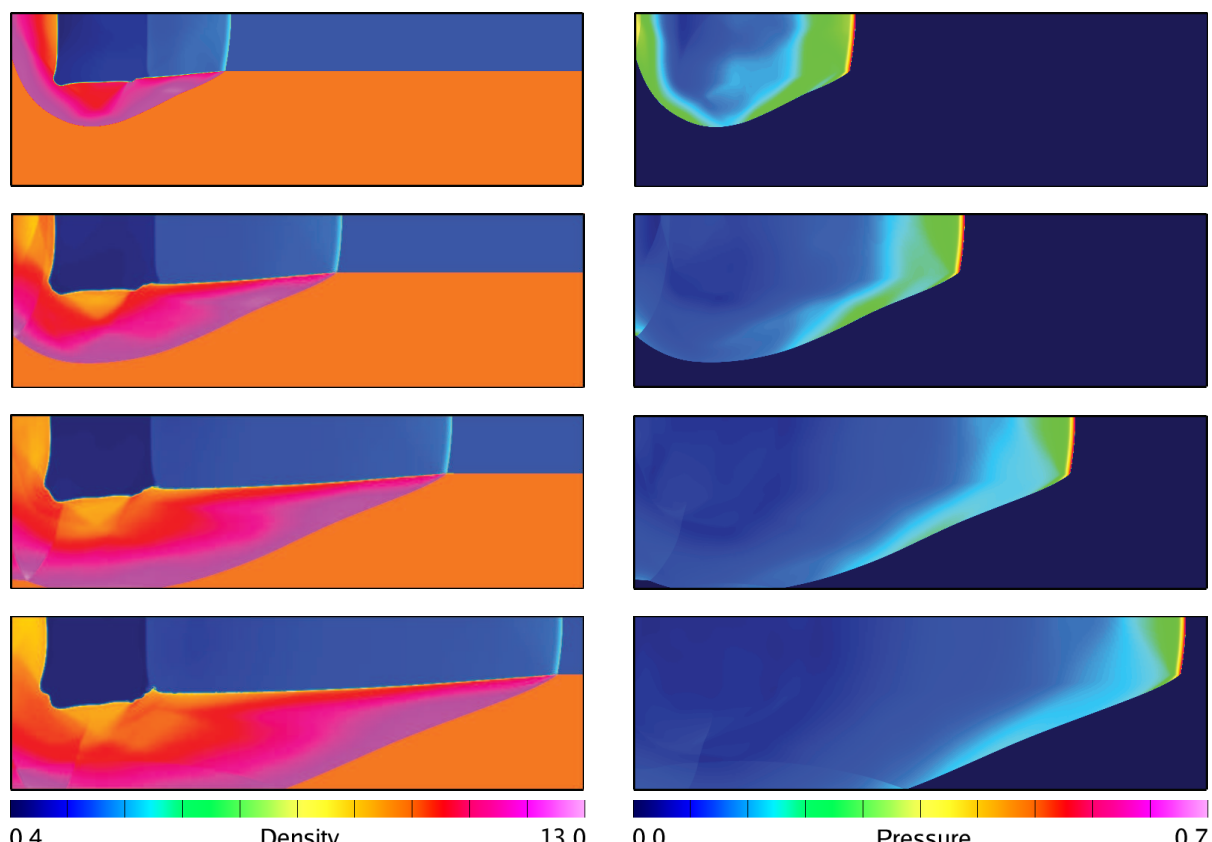

Figure 7: Shaded contours of density (left) and pressure (right) for a strongly confined rate stick at times $t=5,10,15$ and 20 (top to bottom).

the initially square high-pressure booster region expands rapidly, and creates a detonation in the explosive which propagates down the rate stick to the right. The interface between the reactive material and the inert deflects downward behind the detonation, as expected, and as a result the detonation shock becomes curved. As the detonation propagates down the rate stick it approaches a steady state with velocity found to be $D=0.776$, as mentioned earlier, which is slightly lower than the steady planar CJ value given in (34). For convenience of discussion here and elsewhere, we refer to the long-time solution down a rate stick as steady.

An enlarged view of density and pressure near the detonation at $t=20$ is given in figure 8 , and this shows the behavior of the steady wave. On the numerically generated plots of density and pressure, we superimpose the limiting lines for the oblique shocks and the deflected interface from the shock-polar analysis performed earlier. These lines are in good agreement with the computed behavior of the steady wave. We also note that there is no numerical oscillations in pressure near the interface as would be the case without the energy-correction discussed in Section 3.3.

Figure 9 shows the behavior of the density, pressure and reaction progress along the symmetry line, $x_{2}=0$, for the steady solution at $t=20$, and these profiles (red dotted curves) are compared with the corresponding ones for a steady planar ZND wave (black solid curves). Since the detonation shock is very strong so that the peak density is very close to its limiting value, there is little difference in the profiles of density whereas the main difference is seen in the profiles of pressure. Here, the slightly lower detonation speed results in a lower peak pressure, and corresponding lower pressure in the reaction zone, for the strongly confined case. The reaction zone thickness along the line of symmetry is found to be 0.25 approximately, which is larger than the corresponding value of 0.2215 for the steady planar wave because the steady detonation is slightly weaker for this compliant case.

\subsubsection{Weak confinement}

A numerical solution of detonation initiation and propagation in a weakly confined rate stick is obtained using the ambient density and equation of state parameters listed in (36). This solution is shown in figure 10 for times $t=5,10,15$ and 20. For this calculation, we use the same base grid as was used for the strongly confined case, and allow two refined-grid levels on top of the base grid. For the weakly confined case, the 

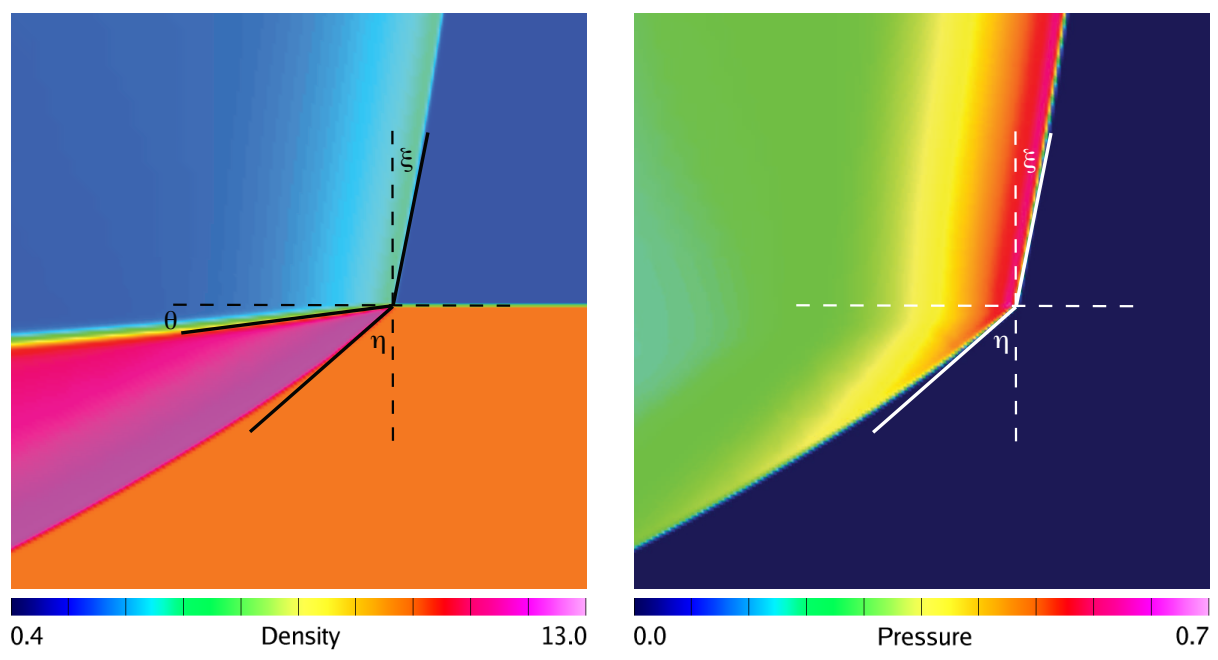

Figure 8: Shaded contours of density (left) and pressure (right) for a strongly confined rate stick at time $t=20$. Asymptotic behavior of the detonation and inert shocks at angles $\xi$ and $\eta$, respectively, and the interface deflection at an angle $\theta$ are shown.
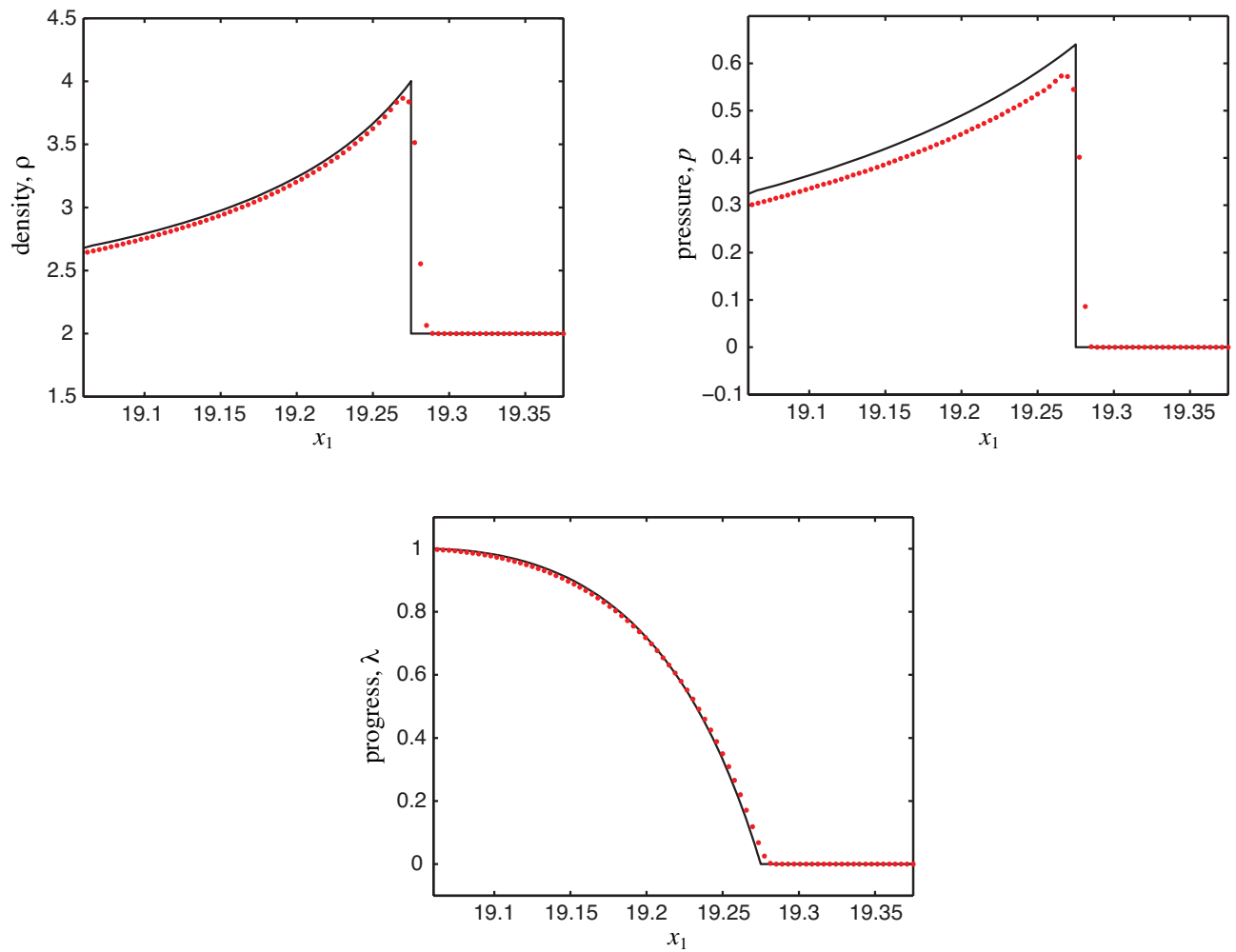

Figure 9: Profiles of density, pressure and reaction progress along $x_{2}=0$ at $t=20$. Profiles for the strong confinement case are given by the dotted-red curves while the profiles for the rigidly confined case are given by the solid-black curves. 

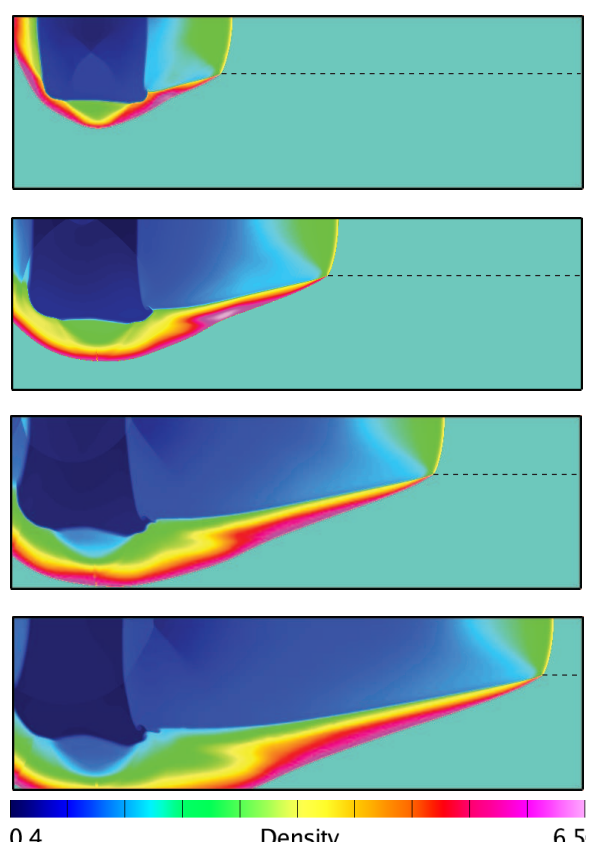

0.4
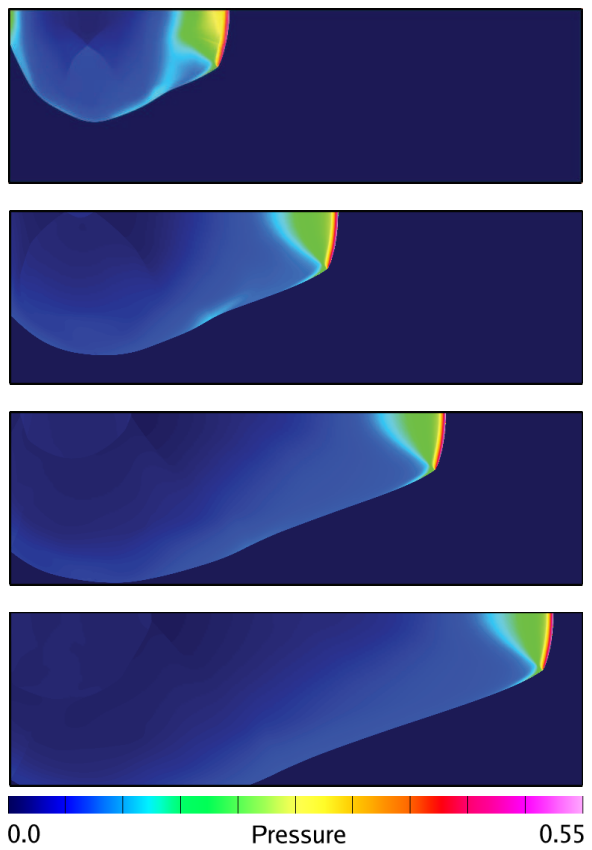

Figure 10: Shaded contours of density (left) and pressure (right) for a weakly confined rate stick at times $t=5,10,15$ and 20 (top to bottom). (The dashed line in each density plot indicates the undisturbed material interface.)

initial expansion of the booster state is noticeably larger, and a detonation is initiated promptly as a result of this stimulus. The downward deflection of the interface behind the detonation is larger for the weakly confined case, and the detonation front is more curved as a result of the greater release behind the detonation near the intersection with the interface. At later times, the detonation approaches a steady state and its velocity along the line of symmetry is found to be $D=0.752$, as was used earlier in the shock-polar analysis.

An enlarged view of the steady detonation structure at $t=20$ is shown in figure 11 and the behavior of $\rho, p$ and $\lambda$ in the reaction zone along $x_{2}=0$ for this solution is shown in figure 12 . In the shaded contour plots of $\rho$ and $p$ in figure 11, we show the limiting lines for the oblique shocks and the deflected interface obtained from the shock-polar analysis, and these are in good agreement with the solution behavior. The expansion in the HE is seen in both plots, but most clearly in the plot of pressure where the limiting Mach lines for the expansion solution are shown. There is good agreement with the local behavior from the shock-polar analysis, as in the strong-confinement case, and the solution behavior is well resolved with no numerical oscillations near the deflected material interface.

The profiles of density, pressure and reaction progress in the reaction zone, shown in figure 12 , for the weakly confined case are similar to the ones shown earlier for the strongly confined case. The peak in the pressure immediately behind the detonation shock is even lower, as expected, due the lower detonation velocity, but the shock is still very strong so there is only a small difference in the profiles of density. The reaction zone length along the line of symmetry is found to be 0.27 , which is larger than the values for the strongly confined and rigidly confined rate sticks.

\subsubsection{Grid convergence}

We now consider the solution of the rate-stick problem for a range of grid resolutions in order to establish the accuracy of the numerical approach. For this study, we use base grids with $\Delta x_{1}=\Delta x_{2}=20 / N$ so that the problem domain shown in figure 4 is spanned by $N$ grid cells in the $x_{1}$ direction on the base level. For each base grid, we use two refined grid levels (with refinement factor equal to 4) so that the effective grid spacing is $1.25 / N$. Figure 13 shows the density and the corresponding AMR grid structure of the solution 

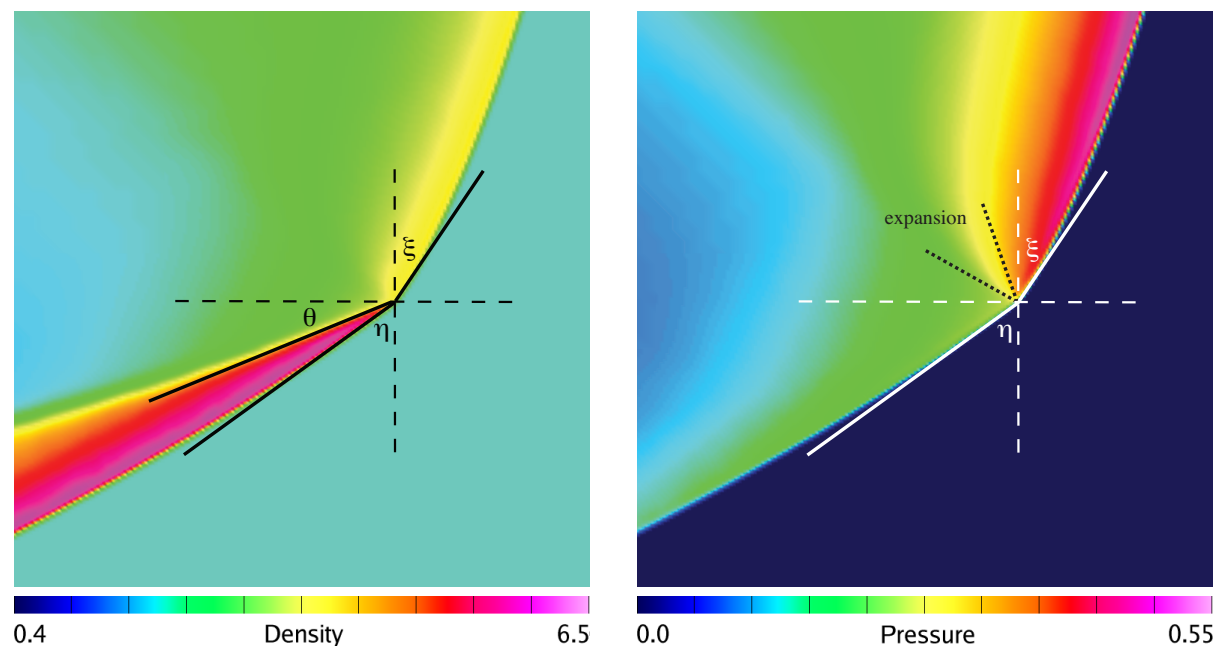

Figure 11: Shaded contours of density (left) and pressure (right) for a weakly confined rate stick at time $t=20$. Asymptotic behavior of the detonation and inert shocks at angles $\xi$ and $\eta$, respectively, and the interface deflection at an angle $\theta$ are shown. The limits of the expansion fan are shown in the plot of pressure.
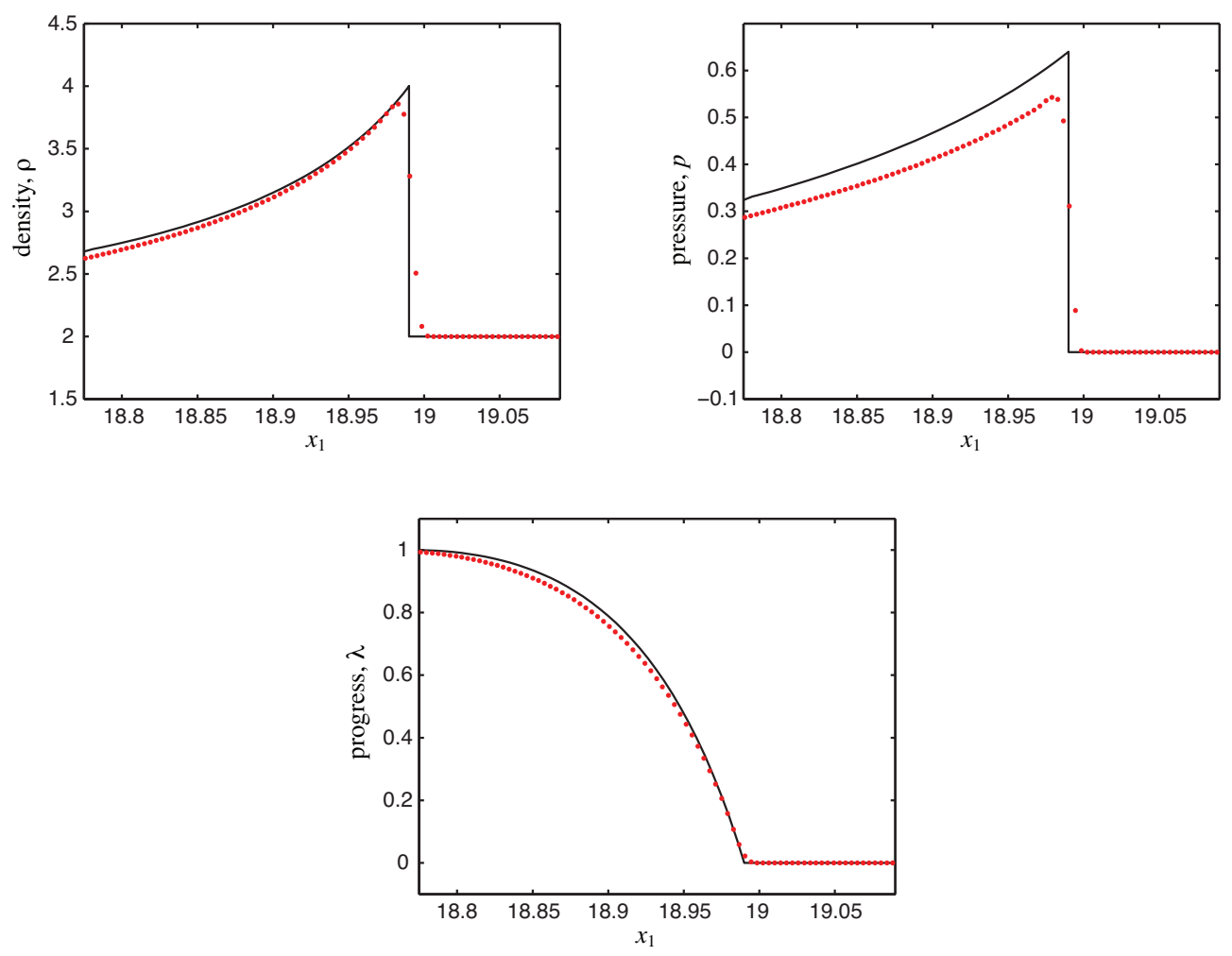

Figure 12: Profiles of density, pressure and reaction progress along $x_{2}=0$ at $t=20$. Profiles for the weak confinement case are given by the dotted-red curves while the profiles for the rigidly confined case are given by the solid-black curves. 

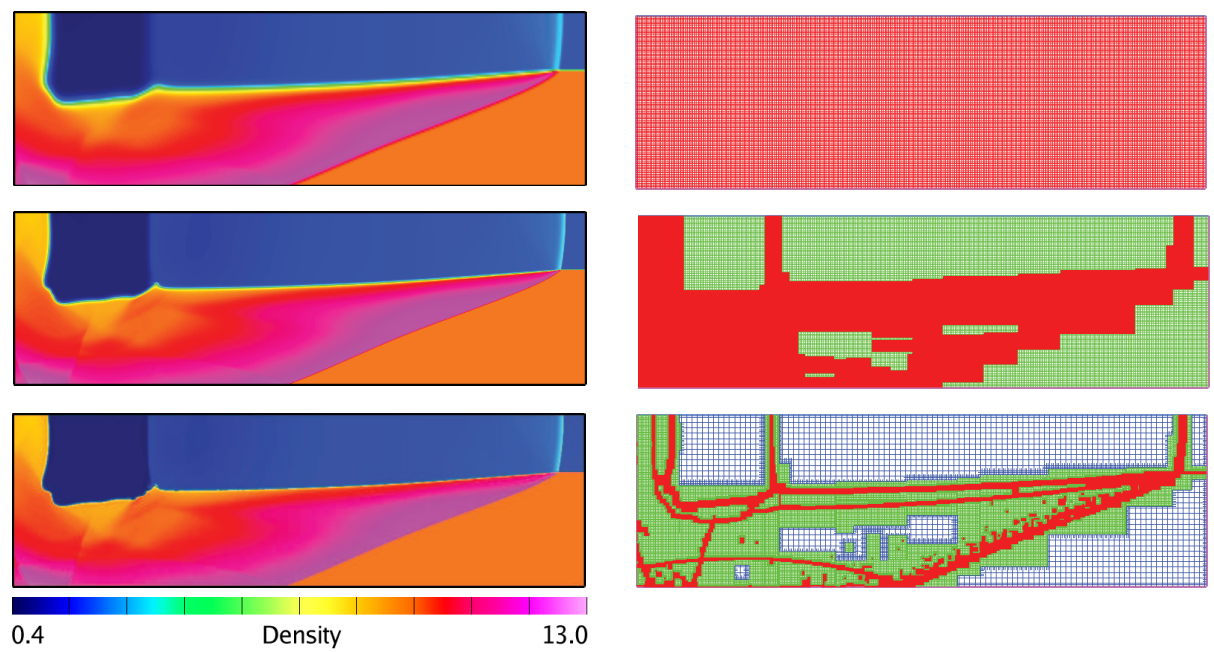

Figure 13: Behavior of the density (left) and corresponding refinement grid structure (right) for a strongly confined rate stick at $t=20$. The grid spacings on the base level are given by $\Delta x_{1}=\Delta x_{2}=20 / N$ with $N=20$ (top), $N=80$ (middle) and $N=320$ (bottom). Two refinement levels are used for each calculation (only a subset of the grid lines are shown for the lower right grid plot).

at $t=20$ for a strongly confined rate stick using $N=20,80$ and 320 . For each grid plot, the base level grid is shown in blue, the level-1 refinement grids in green, and the level-2 refinement grids in red. (Note that the resolution of the refinement grids increase with increasing $N$.) For the $N=20$ case, a single level-2 refinement grid covers the entire domain, and for the $N=80$ case, the solution is represented by refinement grids on levels 1 and 2 alone. For the $N=320$ case, the base grid is fine enough so that the solution is represented using all three grid levels. The general behavior of the solution, as shown by the density, is in general agreement for all three cases considered. Flow features such as the detonation, the deflected interface, and the shock in the inert become sharper as the grid resolution increases. Grid convergence of the solution is apparent in these plots. The results shown previously in Sections 4.1.2 and 4.1.3 correspond to the finest grid resolution with $N=320$.

A more detailed investigation of grid convergence is performed by taking slices of the solution at $t=20$ along the lines $x_{2}=0$ and $x_{1}=18$ for various grid resolutions. The results of this investigation are shown in figure 14 for a sequence of AMR grids with $N=40,80, \ldots, 320$. The line $x_{2}=0$ is chosen to illustrate the convergence of the detonation shock and the reaction zone behind it. The pair of plots on the top row of figure 14 show the behavior of density and pressure, respectively, along this line. As the grid resolution increases, the position of the leading shock of the detonation and the peaks in density and pressure behind it converge nicely so that the behavior of the detonation on the finest grid level is well resolved. The density and pressure along $x_{1}=18$, given in the bottom pair of plots in figure 14, show the behavior across the material interface and the shock in the inert. The plot of density shows good grid resolution of the interface, and the plots of both density and pressure show good resolution of the shock in the inert. The plot of pressure shows smooth behavior in the vicinity of the material interface which provides further evidence of the effectiveness of the energy-corrected Godunov scheme.

A similar convergence study is performed for a weakly confined rate stick and the results are shown in figures 15 and 16. The grid resolutions chosen for this case are the same as those for the strongly confined case. Figures 15 shows the behavior of density and the corresponding grids at $t=20$ and here, as before, we note that the solution on the finest level is nicely represented with sharp features of the solution. The behavior of density and pressure along the lines $x_{2}=0$ and $x_{1}=18$, shown in figure 16, indicate the convergence. The detonation along the line $x_{2}=0$ is well resolved at the finest grid level as are the material interface and the shock in the inert along the line $x_{1}=18$. 

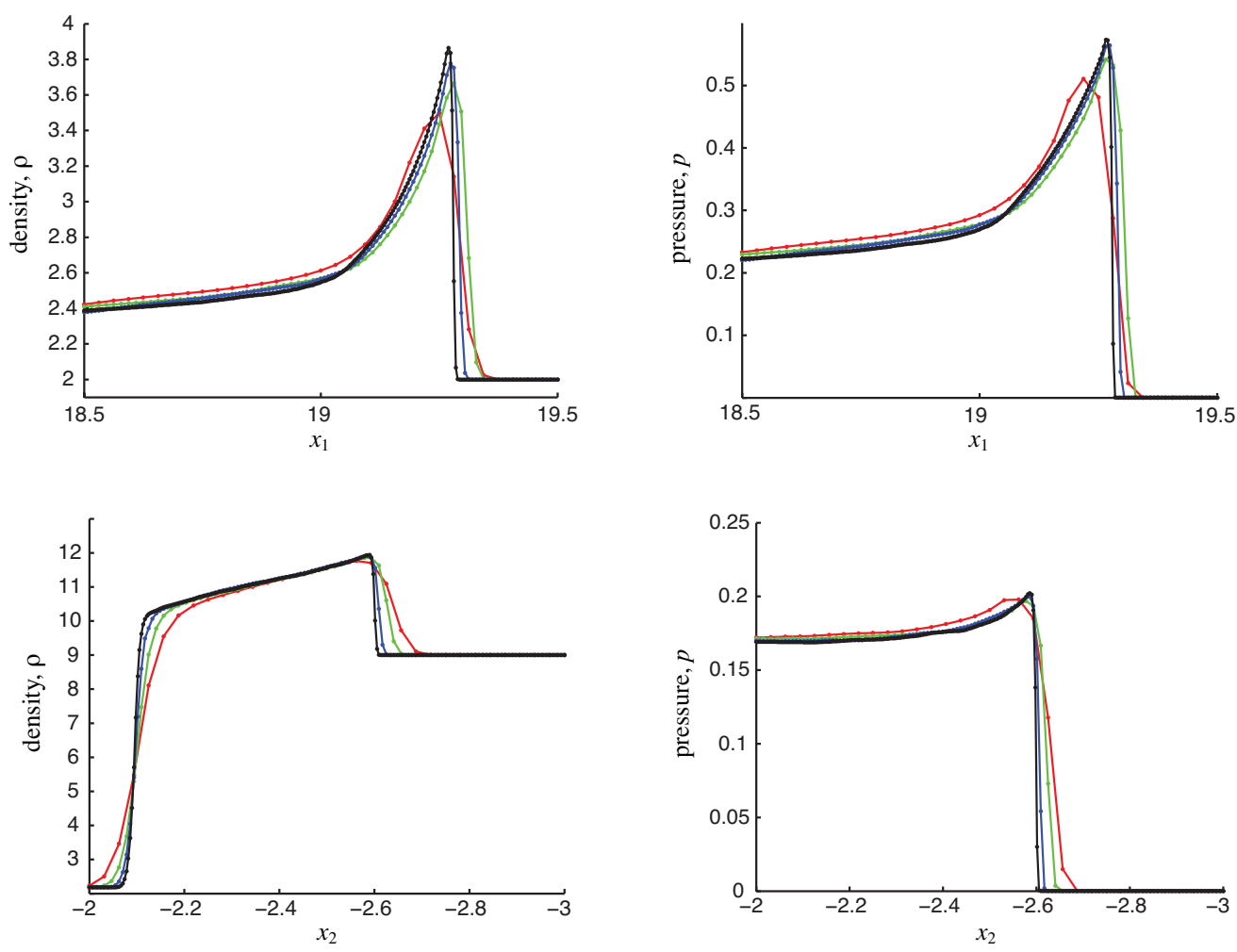

Figure 14: Behavior of density (left) and pressure (right) along lines $x_{2}=0$ (top) and $x_{1}=18$ (bottom) for a strongly confined rate stick. Solutions correspond to AMR grids with $N=40$ (red), $N=80$ (green), $N=160$ (blue) and $N=320$ (black).
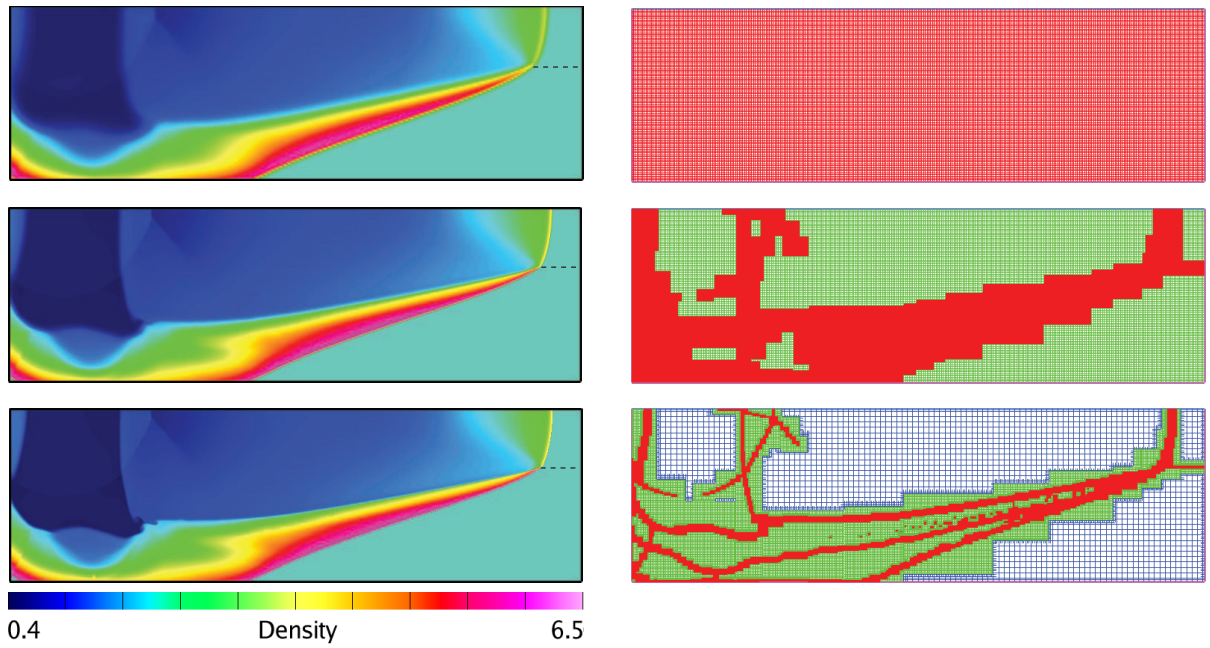

Figure 15: Behavior of the density (left) and corresponding refinement grid structure (right) for a weakly confined rate stick at $t=20$. The grid spacings on the base level are given by $\Delta x_{1}=\Delta x_{2}=20 / N$ with $N=20$ (top), $N=80$ (middle) and $N=320$ (bottom). Two refinement levels are used for each calculation (only a subset of the grid lines are shown for the lower right grid plot). 

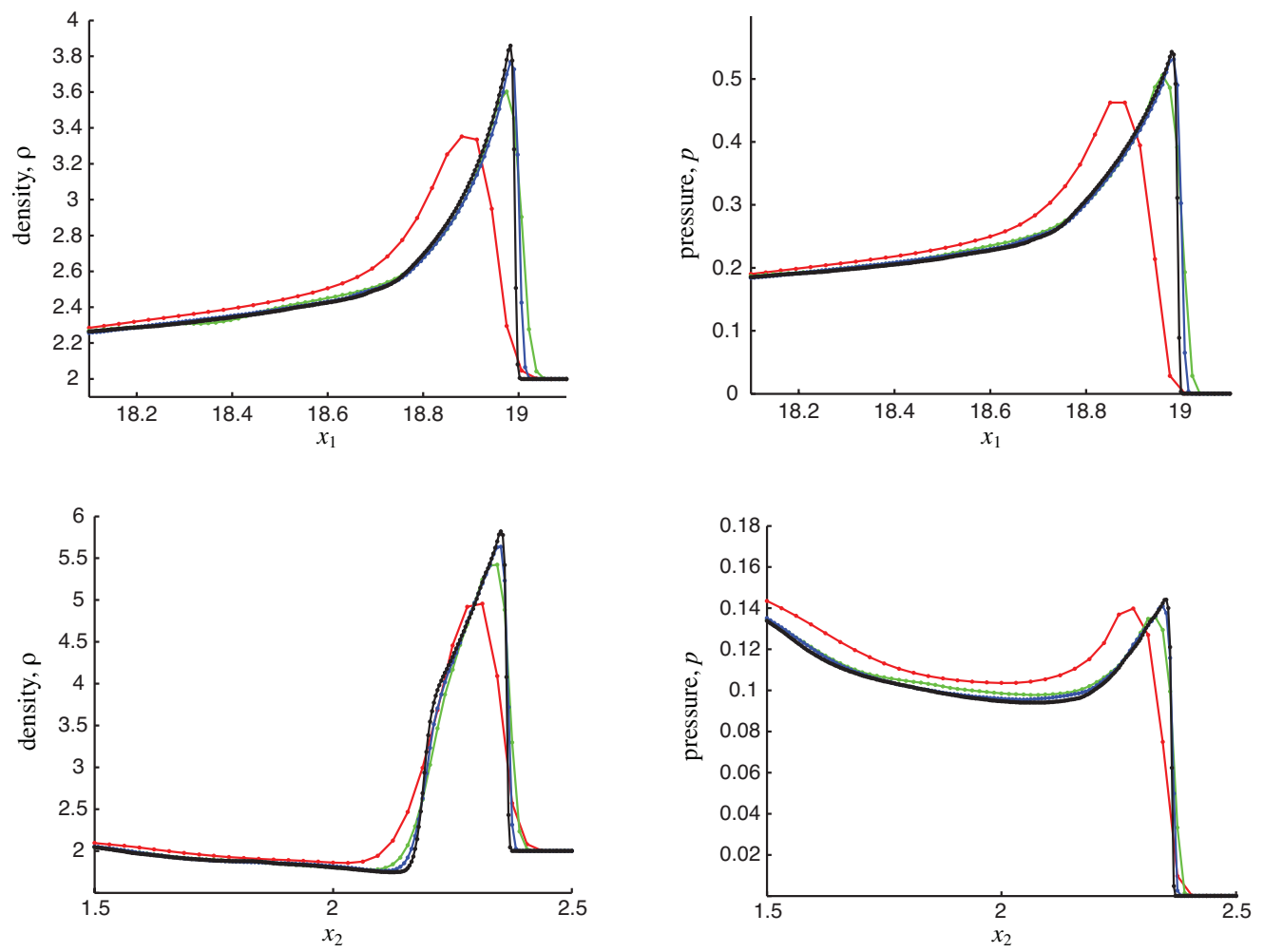

Figure 16: Behavior of density (left) and pressure (right) along lines $x_{2}=0$ (top) and $x_{1}=18$ (bottom) for a weakly confined rate stick. Solutions correspond to AMR grids with $N=40$ (red), $N=80$ (green), $N=160$ (blue) and $N=320$ (black). 


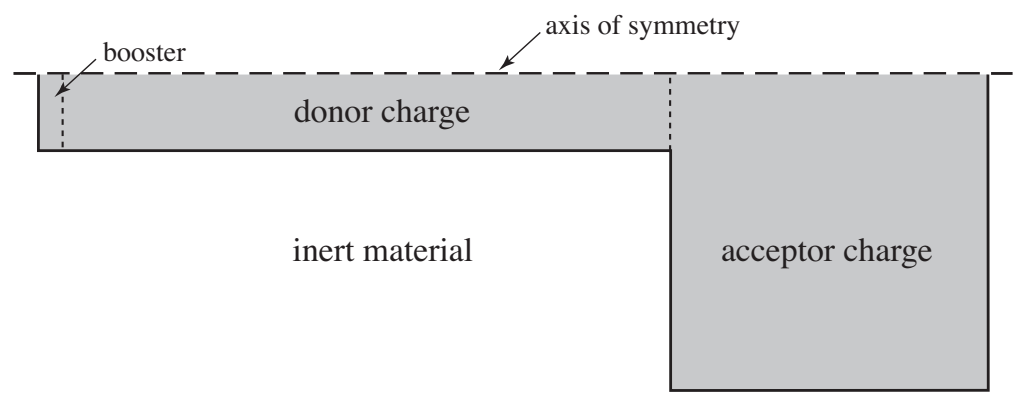

Figure 17: Schematic representation of the corner-turning geometry.

\subsubsection{Initial conditions for $\lambda$ in the inert material}

In regions of the flow where there is no reactive material present, $\phi_{r}=0$ and the value for $\lambda$ plays no role in the exact solution. Thus, for a flow in which a sharp interface (a contact discontinuity) separates the reactive material from the inert, the choice for $\lambda$ in the initial state of the inert need not be specified. For our numerical treatment of the governing equations, we require an initial value for $\lambda$ for all grid points, including those for which $\phi_{r}=0$. A first choice might be to set $\lambda=1$ where $\phi_{r}=0$ so that the reaction rate is zero at such points and no integration of the reaction rate is carried out where it is not needed. The problem with this choice, however, is that numerical diffusion, while small, plays a role near the material interface. If $\lambda$ is set to 1 initially on the inert side of the material interface while an accurate value for $\lambda$ between 0 and 1 in the reaction zone is required on the reactive side, then numerical diffusion would have the effect of artificially increasing $\lambda$ in the reaction zone near the material interface. As there is heat release associated with an increase in $\lambda$, an additional amount of energy would be released into the system near the material interface due to numerical diffusion there. In order to avoid this situation, we choose instead to set $\lambda=0$ initially where $\phi_{r}=0$ as noted earlier. For this choice, the confiner is reactive but thermoneutral. Since change in $\lambda$ along particle paths is controlled by the reaction rate law $\mathcal{R}$ throughout the entire domain, and since pressure is continuous across the material interface, $\lambda$ is continuous there as well and hence the effect of numerical diffusion of $\lambda$ is minimal. A disadvantage of this choice is that the reaction rate is computed everywhere, including in the inert, but we accept this extra numerical cost in order to disarm any potential problems arising from heat release due to numerical diffusion near the material interface.

\subsection{Detonation diffraction at a $90^{\circ}$ corner}

Corner-turning calculations are performed using the ignition-and-growth (IG) model for both strong and weak confinements. The geometry of the corner-turning problem considered is shown in figure 17 . The explosive consists of two cylindrical rate sticks, a donor charge on the left and an acceptor charge on the right with diameters taken to be $12 \mathrm{~mm}$ and $50 \mathrm{~mm}$, respectively. A detonation is initiated by a highpressure booster state on the left end of the donor charge. The resulting detonation travels axially along the donor charge and diffracts at the $90^{\circ}$ corner at the junction of the donor and acceptor charges. The length of the donor charge, taken to be $50 \mathrm{~mm}$, is long enough so that the detonation wave becomes steady prior to its passage into the acceptor charge. The geometry used for this problem is motivated by recent experiments for the explosive PBX 9502 discussed in [13]. Our main focus in this section is to describe the diffraction behavior for both strong and weak confinements using the numerical method developed in this paper. For the strong-confinement case, we also compare the results with analogous calculations for a rigid confinement following our recent work in [11]. Of particular interest in these calculations is whether the detonation fails, and to what extent the behavior depends on the strength of the confinement.

The calculations are performed for the IG model which consists of the governing equations in (4) with the mixture JWL equation of state (EOS) described in Section 2.1 and the multi-stage reaction rate given in (13). The EOS and rate parameters are taken from [6] which are calibrated for PBX 9502 (95\% TATB, $5 \% \mathrm{KelF}$ ) at $25^{\circ} \mathrm{C}$. The density of the undisturbed solid explosive is $\rho_{0}=1895 \mathrm{~kg} / \mathrm{m}^{3}$ and following the 
analysis in [11], the velocity of a steady, one-dimensional CJ detonation is found to be $D_{\mathrm{CJ}}=7.7161 \mathrm{~km} / \mathrm{s}$. These values are used to define the following reference scales:

$$
\rho_{\text {ref }}=\rho_{0}, \quad u_{\text {ref }}=D_{\mathrm{CJ}}, \quad p_{\text {ref }}=\rho_{\text {ref }} D_{\mathrm{CJ}}^{2}, \quad E_{\text {ref }}=D_{\mathrm{CJ}}^{2} .
$$

The reference scale for time and length are taken to be

$$
t_{\mathrm{ref}}=1 \mu \mathrm{s}, \quad x_{\mathrm{ref}}=u_{\mathrm{ref}} t_{\mathrm{ref}}=7.7161 \mathrm{~mm} .
$$

Using the reference scales defined above, the dimensionless EOS parameters are collected in table 1. Included in the table are dimensionless EOS parameters for both strong and weak inert confinements. These parameters are chosen to yield shock-polar diagrams, shown in figure 18, which are similar to those for the strong and weak confinement cases discussed previously for the rate-stick problem and shown in figures $5(\mathrm{~b})$ and $6(\mathrm{~b})$, respectively. For the strong case, a solution is found with

$$
\xi=0.272, \quad \eta=0.380, \quad \theta=0.120, \quad p=.301,
$$

while the solution for the weak case is given by

$$
\xi=\xi_{\mathrm{s}}=0.797, \quad \eta=1.24, \quad \theta=0.179, \quad p=0.0581 .
$$

Finally, the dimensionless ignition-and-growth rate parameters are given in table 2.

\begin{tabular}{|c|c|c|c|c|c|}
\hline \multicolumn{2}{|l|}{$\mathcal{R}_{I}$} & \multicolumn{2}{|l|}{$\mathcal{R}_{G_{1}}$} & \multicolumn{2}{|c|}{$\mathcal{R}_{G_{2}}$} \\
\hline Parameter & Value & Parameter & Value & Parameter & Value \\
\hline$I$ & $4.0 \mathrm{e} 6$ & $G_{1}$ & 1400. & $G_{2}$ & 33.85 \\
\hline$b$ & 0.667 & $c$ & 0.667 & $e$ & 0.667 \\
\hline$a$ & 0.214 & $d$ & 1 & $g$ & 0.667 \\
\hline$x$ & 7 & $y$ & 2 & $z$ & 1 \\
\hline$\lambda_{\text {ig,max }}$ & 0.025 & $\lambda_{G_{1}, \max }$ & 0.8 & $\lambda_{G_{2}, \min }$ & 0.8 \\
\hline
\end{tabular}

\begin{tabular}{|c|c|c|c|c|}
\hline \hline Parameter & Solid, $s$ & Products, $g$ & Inert, $i$ (strong) & Inert, $i$ (weak) \\
\hline \hline$A_{k}$ & 560.2 & 12.07 & 100.0 & 40.0 \\
\hline$B_{k}$ & -0.03964 & 0.6381 & -0.04 & 0.5 \\
\hline$R_{1, k}$ & 11.3 & 6.2 & 10.0 & 20.0 \\
\hline$R_{2, k}$ & 1.13 & 2.2 & 1.5 & 5.0 \\
\hline$\omega_{k}$ & 0.8938 & 0.5 & 0.8 & 0.4 \\
\hline$C_{v, k}$ & 1.0 & 0.4021 & 1.0 & 1.0 \\
\hline$Q_{k}$ & 0.06116 & 0 & 0.06116 & 0.06116 \\
\hline$v_{0, k}$ & 1.0 & 5.0 & 1.0 & 1.0 \\
\hline \hline
\end{tabular}

Table 1: Equation-of-state parameters for PBX 9502, both solid explosive and gaseous products, and for strong and weak inert confinements.

Table 2: Ignition-and-growth rate law parameters for PBX 9502.

The calculations for strong and weak confinements are performed using a Cartesian base grid covering the domain $-6.48 \leq x_{1} \leq 3.24$ and $-3.24 \leq x_{2} \leq 0$ (corresponding to a dimensional $x_{1}$ between $-50 \mathrm{~mm}$ and $25 \mathrm{~mm}$ and a dimensional $x_{2}$ between $-25 \mathrm{~mm}$ and 0 ), and using two refinement grid levels. The axis of symmetry is $x_{2}=0$. The ambient state of the reactive material in the system at $t=0$ is

$$
\rho=1, \quad u_{1}=u_{2}=0, \quad p=0, \quad \lambda=0, \quad \phi_{r}=1
$$



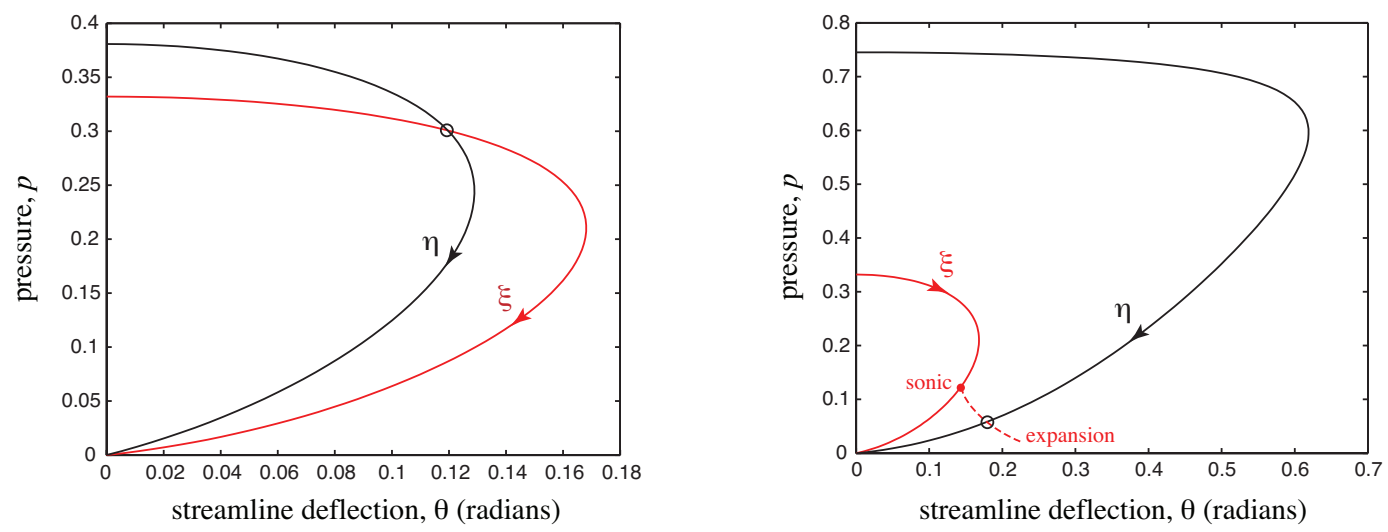

Figure 18: Shock-polar curves for the HE (red) and inert (black) using the EOS parameters in table 1. The left plot is for a strong confinement and the right plot for a weak confinement. The weak case includes a Prandtl-Meyer expansion curve for the HE (dashed red curve).

for $-6.48 \leq x_{1} \leq 0,-0.778 \leq x_{2} \leq 0$ (donor charge) and for $0 \leq x_{1} \leq 3.24,-3.24 \leq x_{2} \leq 0$ (acceptor charge). The state of the inert material in the remainder of the flow domain at $t=0$ is

$$
\rho=\rho_{0, \text { inert }}, \quad u_{1}=u_{2}=0, \quad p=0, \quad \lambda=0, \quad \phi_{r}=0,
$$

where $\rho_{0, \text { inert }}=1.3$ for the strong confinement and $\rho_{0 \text {,inert }}=1.0$ for the weak confinement. A detonation is initiated by introducing a small high-pressure region with $p=0.24$ and $\lambda=1$ near the left end of the donor charge at $t=0$. The high pressure creates a shock wave which propagates axially to the right in the reactive material and radially outward into the inert. The shock quickly transitions into a detonation in the reactive material which propagates axially down the donor charge. The donor charge is sufficiently long (approximately 4 donor-charge diameters) so that the detonation achieves a steady structure by the time it reaches the acceptor charge at $x_{1}=0$. Our primary interest is the behavior of the detonation once it reaches the acceptor charge, and these results are discussed in Sections 4.2.1 and 4.2.2 below for the cases of strong and weak confinement, respectively. Comparisons are also made with results for a rigid confinement in which the interface between the reactive material and the inert at $t=0$ is replaced by a fixed-grid boundary where a slip-wall boundary condition is applied.

\subsubsection{Strong confinement}

The numerical results for the strong-confinement case are shown in figures 19, 20 and 21. Figure 19 displays results at early times just before and after the detonation meets the the corner. The top frames in the figure show a numerical schlieren $\sigma$, the pressure $p$, and reaction progress $\lambda$ at $t=6.5$ when the steady detonation in the donor charge has almost reached the corner. The schlieren is a gray-scale image of

$$
\sigma=\exp \left\{-\beta\left(\frac{|\nabla \rho|-\min |\nabla \rho|}{\max |\nabla \rho|-\min |\nabla \rho|}\right)\right\},
$$

where the minimum and maximum are taken over the entire domain, and $\beta$ is the exposure (taken to be 15). The range of $\sigma$ plotted is 0.3 to 1 where the lower limit is assigned to black and the upper limit is assigned to white in the gray scale image. The axis of symmetry is along the top of each frame, but the view is enlarged to illustrate the behavior near the corner and so the full radial extent of the acceptor charge is not shown. The schlieren shows the steady curved detonation wave, the deflected interface separating the reaction products and the inert, and the transmitted shock in the inert. The plot of pressure shows the peak in pressure behind the detonation, which is highest near the axis of symmetry. The plot of reaction progress indicates the location and behavior of the reaction zone. This variable is relevant only for the reactive 
material and so its behavior in regions of the flow where $\phi_{r}<0.5$ is not shown to avoid unnecessary distraction. The bottom three frames in figure 19 show the early post-diffraction behavior. Here, we observe that the leading shock of the detonation traveling down the left side of the acceptor charge is composed of a curved segment from the diffraction at the corner and a nearly planar (conical, accounting for the axisymmetric geometry) segment which has passed from the inert and into the explosive. The shock in this region of the flow is weaker, as expected, and the reaction zone is wider, as indicated by the plots of pressure and reaction progress.
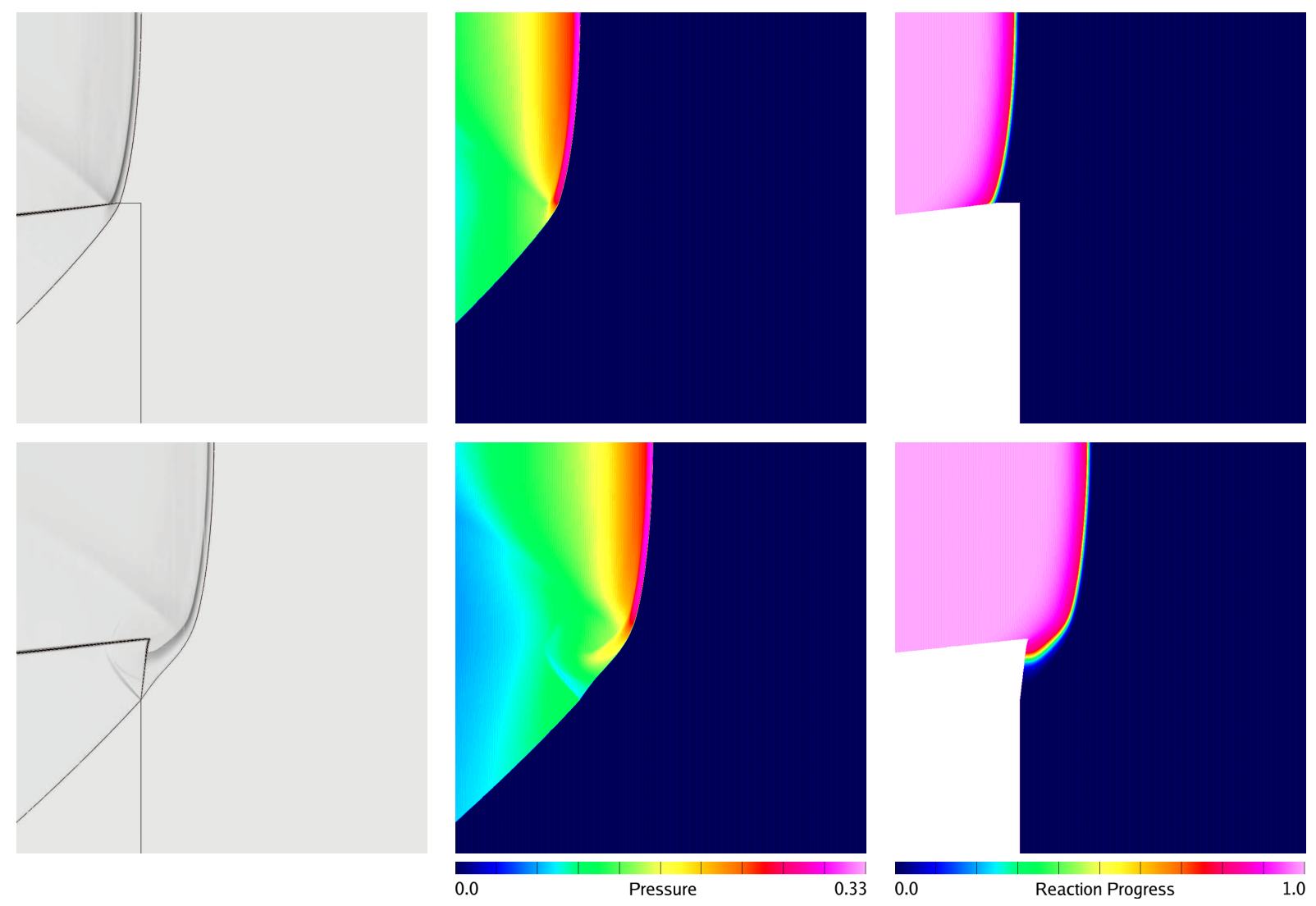

Figure 19: Strong confinement: schlieren (left), pressure (middle) and reaction progress (right) at $t=6.5$ (top frames) and $t=6.8$ (bottom frames).

While the weakening of the leading shock and a corresponding lengthening of the reaction zone behind it hint at a possible failure of the detonation, no such behavior is observed for the strong-confinement case. The density behind the leading shock never drops below the threshold, $\rho_{\text {ign }}=1+a$, for the ignition stage of the IG rate law in (13), and thus the reaction rate is never turned off completely. As a result, we observe in figure 20 that reaction, while weakened temporarily, ultimately strengthens leading to the re-formation of a secondary detonation behind the leading shock which travels radially outwards along the left face of the acceptor charge. This behavior is seen at the times $t=7.0$ and 7.2 shown in the figure. The conical shock which has passed from the inert into the explosive is relatively weak and now acts to pre-condition the explosive ahead of the secondary detonation.

The frames in figure 21 show a broader view of the behavior at the later time $t=8.1$. For this view, the axis of symmetry remains at the top of each frame, but the radial extent displayed is larger than that in the frames in the previous two figures. Here, we note that the secondary detonation has overtaken the conical shock and become part of an overall, expanding, curved detonation. There is little evidence of the local behavior from the diffraction at the corner, and there is no region of unreacted material left behind the expanding detonation. 

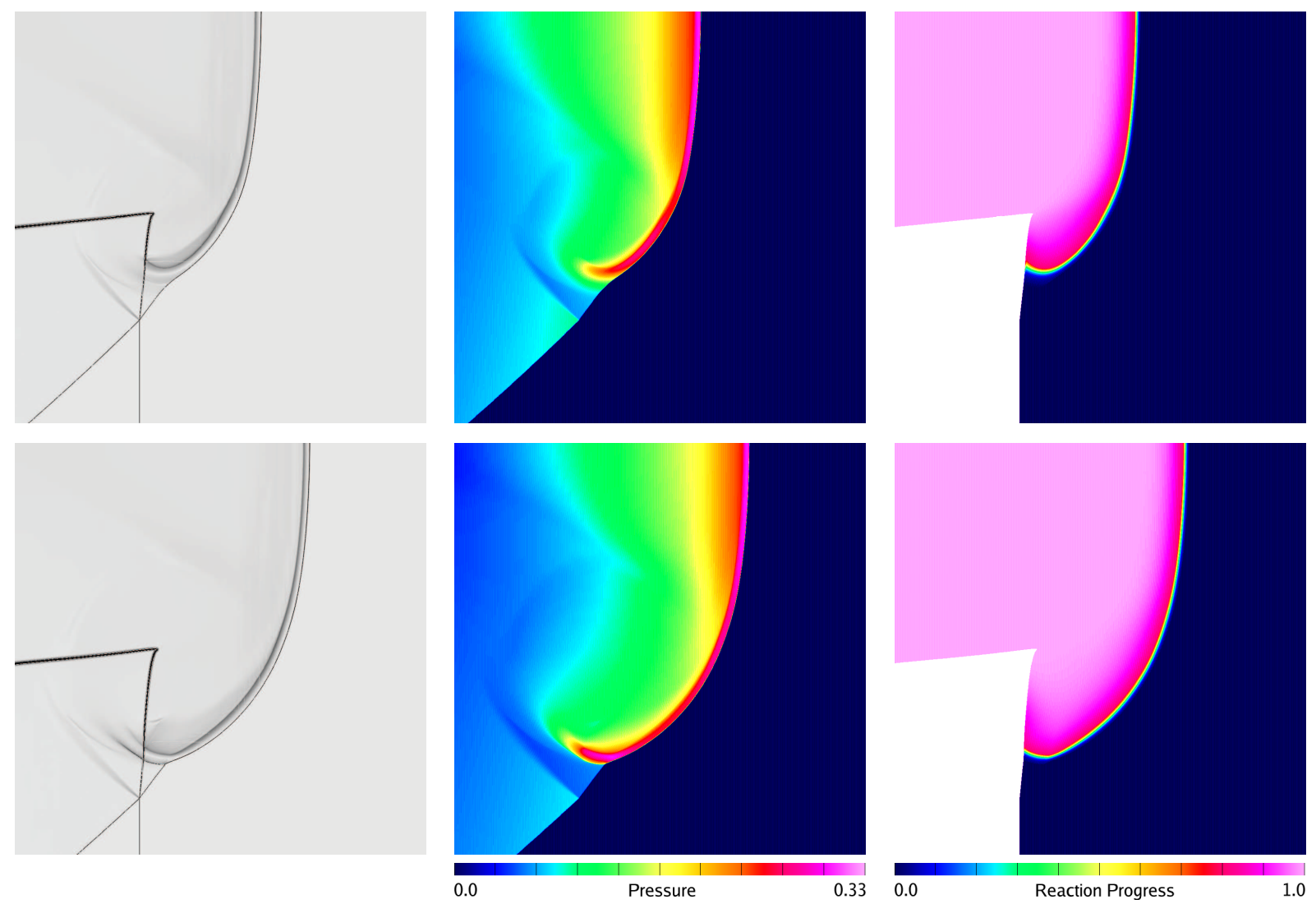

Figure 20: Strong confinement: schlieren (left), pressure (middle) and reaction progress (right) at $t=7.0$ (top frames) and $t=7.2$ (bottom frames).
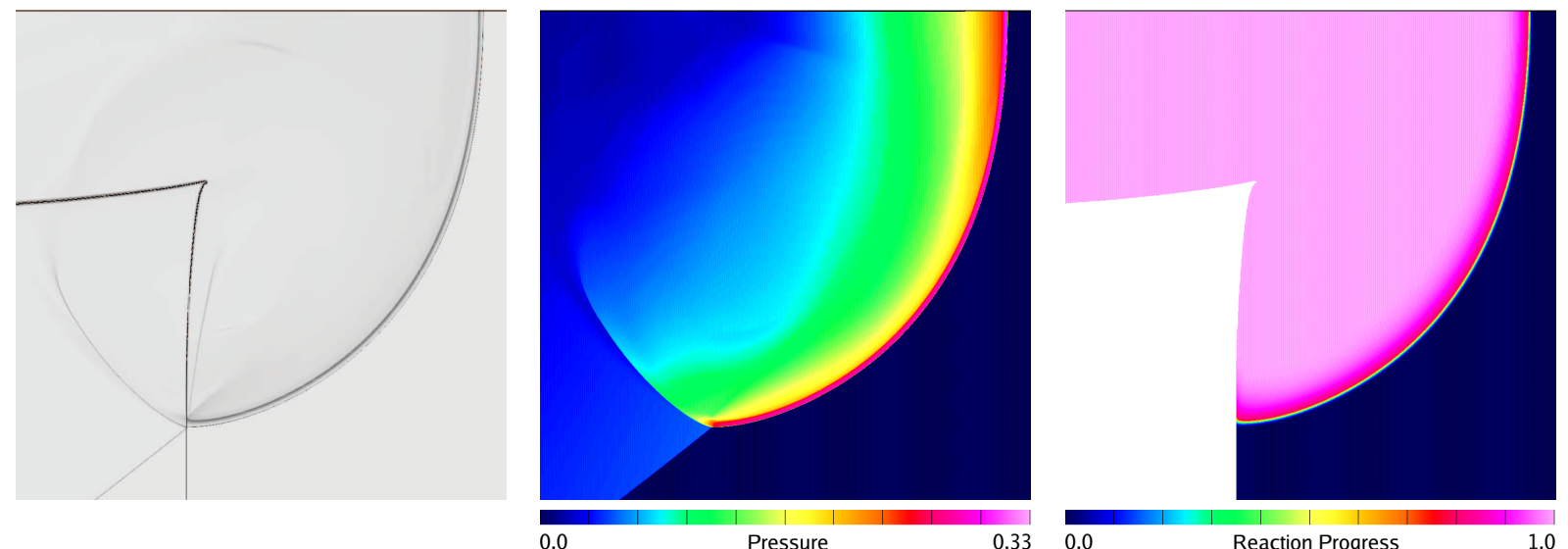

Figure 21: Strong confinement: schlieren (left), pressure (middle) and reaction progress (right) at $t=8.1$. 
For the case of a strong confinement, it is interesting to the compare the results with those given by a rigid confinement. Figure 22 shows $\sigma, p$ and $\lambda$ at $t=6.7,6.9$ and 7.1 for detonation diffraction at a rigid $90^{\circ}$ corner. The geometry of the donor-acceptor charge system for the rigid-boundary calculation is the same as the strong-confinement case except that the corner is rounded slightly for the rigid case. The radius of the smoothed corner is very small, approximately the width of the CJ-detonation reaction zone, and is employed, following the work in [11], to regularize the singularity that would occur at a sharp rigid corner. As discussed in [11], the rounded corner has a negligible effect on the diffraction behavior once the detonation has travelled a few reaction-zone lengths past the corner. The top-row frames at $t=6.7$ for the rigid case may be compared with the frames at $t=6.8$ in figure 19 for the strong-confinement case. In both cases, there is a small separation of the leading shock of the detonation and the reaction zone behind it, but this separation is short lived as the reaction strengths behind the leading shock. In the strong-confinement case, this strengthening leads to the formation of a secondary detonation, as mentioned earlier, while in the rigid case the reaction zone simply strengthens and re-attaches to the leading shock (see frames at $t=6.9$ and 7.1 in figure 22). There is no conical shock from the inert in the rigid case, but the long-time behavior is similar for the two cases. The detonation develops into an expanding, curved wave with no unreacted explosive left behind.

\subsubsection{Weak confinement}

Numerical results for the weak-confinement case are displayed in figures 23, 24 and 25. The top frames of figure 23 show the schlieren, pressure and reaction progress at a time, $t=6.7$, when the detonation from the donor charge has just reached the acceptor charge. The detonation is noticeably more curved than that for the strong-confinement case, and the interface deflection is larger. The thickness of the reaction zone behind the leading shock near the interface is also larger indicating a weaker detonation there. The bottom frames in the figure show the behavior at a short time later, $t=7.3$, when the detonation has passed into the acceptor charge. The leading shock of the detonation has turned the corner and weakened, and the pressure behind it is lower than was seen previously in the strong-confinement case. (The shaded contour scale of pressure used here is the same as in the strong-confinement figures.) This is due to the greater lateral release of the interface separating the left side of the acceptor charge and the inert. As a result, the separation between the leading shock and the reaction behind it is larger, and a region of fully unreacted material behind the lead shock is seen clearly in the plot of $\lambda$.

The solution at later times shows a strengthening of the reaction in a region of the flow away from the interface, and then a lateral movement of a resulting secondary detonation towards the interface. This behavior is shown in figure 24. The top frames in the figure at $t=7.7$ show the early behavior of this strengthening reaction. In the plot of pressure at this time, for example, we note the formation of a highpressure "hook." This feature is created by an increased rate of reaction behind the diffracted lead shock, away from the interface where the shock is not as weak. Here the post-shock density is high enough to overcome the ignition threshold so that a strong reaction can develop. The high pressure leads to the formation of a shock and a secondary detonation, which propagates laterally towards the interface to consume the unreacted material there. Once the detonation reaches the interface, as seen in the bottom frames of figure 24 at $t=7.9$, its lead shock passes into the inert material and the detonation propagates radially inwards towards the axis of symmetry to consume the remaining unreacted material.

A broader view of the flow at $t=8.5$ is shown in figure 25. In the schlieren plot and in the plot of pressure, we see that an expanding detonation is fully established, and in the plot of reaction progress we observe that the unreacted material previously left behind the diffracted shock is now nearly all consumed.

We note that the overall behavior for this weak-confinement case is similar to that for a rigidly confined "hockey-puck" calculation described in [11], and motivated in part by experiments and computations for the explosive LX-17 reported in [10]. While the parameters for the IG model used in [11] for LX-17 differ from those used here for PBX-9502, the two explosives are similar and a similar behavior was observed. For the hockey-puck geometry, the leading shock of the expanding spherical detonation was weakened by the diffraction at a $90^{\circ}$ corner, and the reaction zone behind it separated resulting in a region of unreacted material behind the weakened shock. At a later time, a high-pressure hook formed and a secondary detonation was established which moved laterally towards the rigid boundary to consume the unreacted material. Thus, 

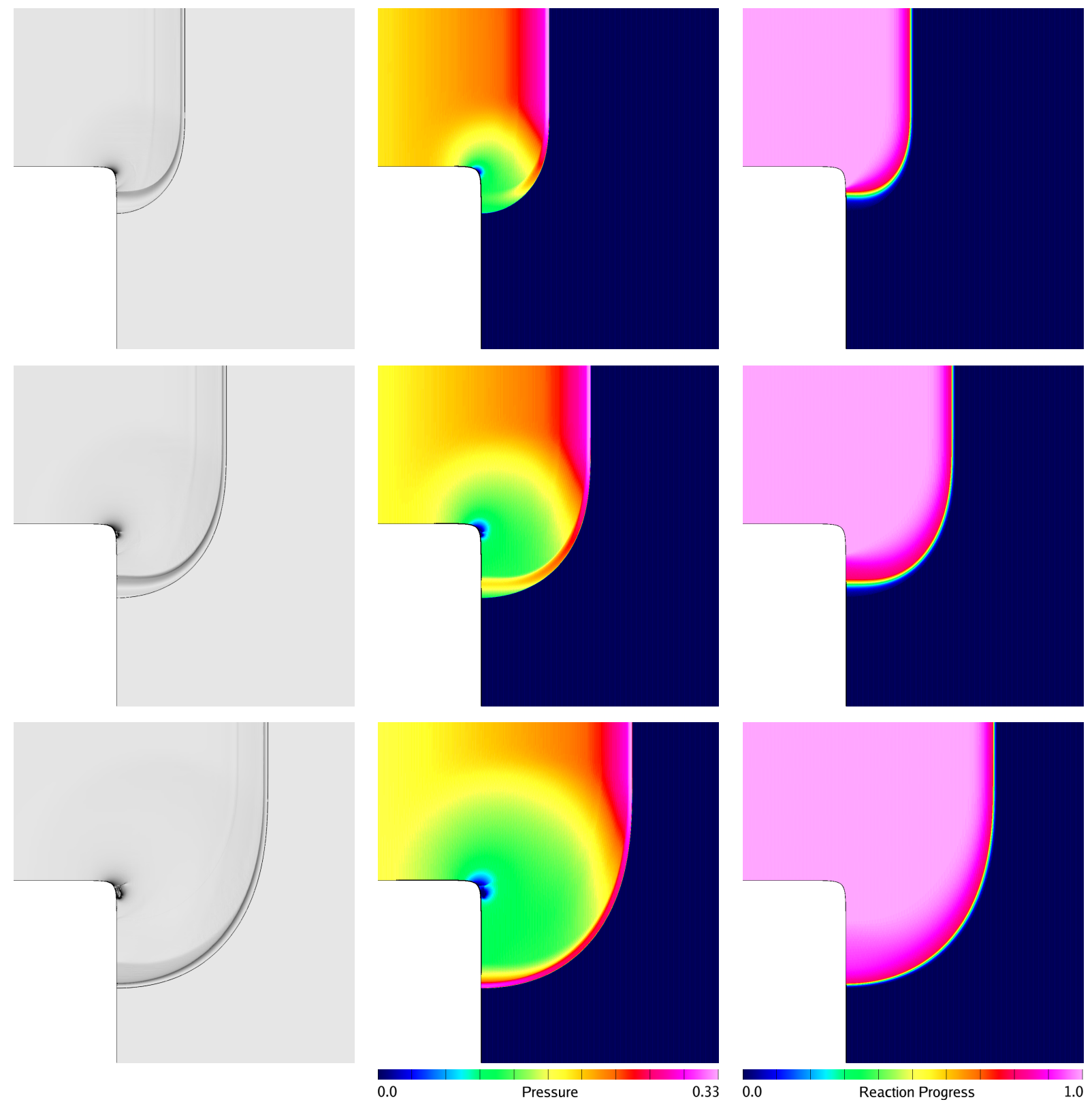

Figure 22: Rigid confinement: schlieren (left), pressure (middle) and reaction progress (right) at $t=6.7$, 6.9 and 7.1 (top to bottom). 

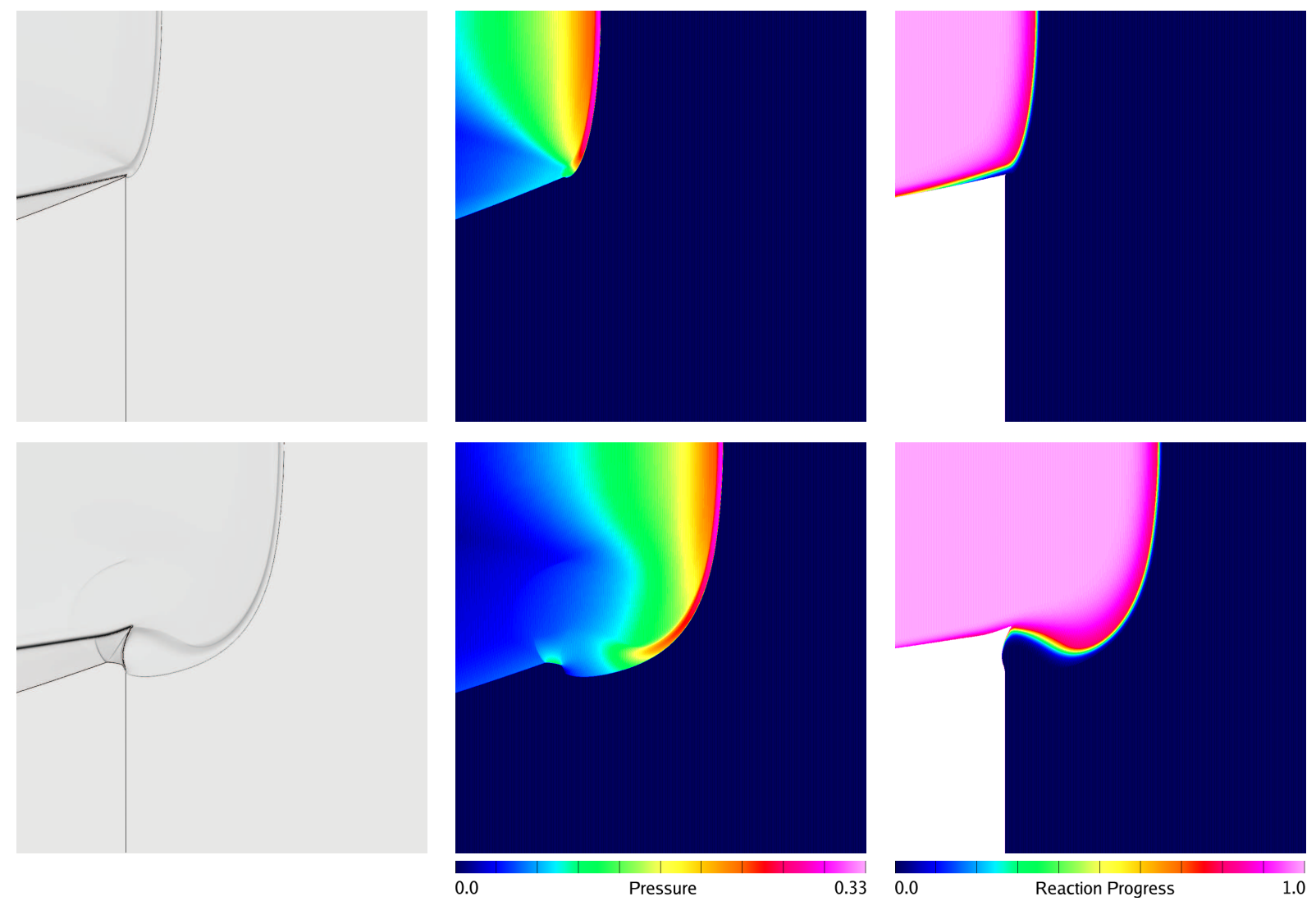

Figure 23: Weak confinement: schlieren (left), pressure (middle) and reaction progress (right) at $t=6.7$ (top frames) and $t=7.3$ (bottom frames). 

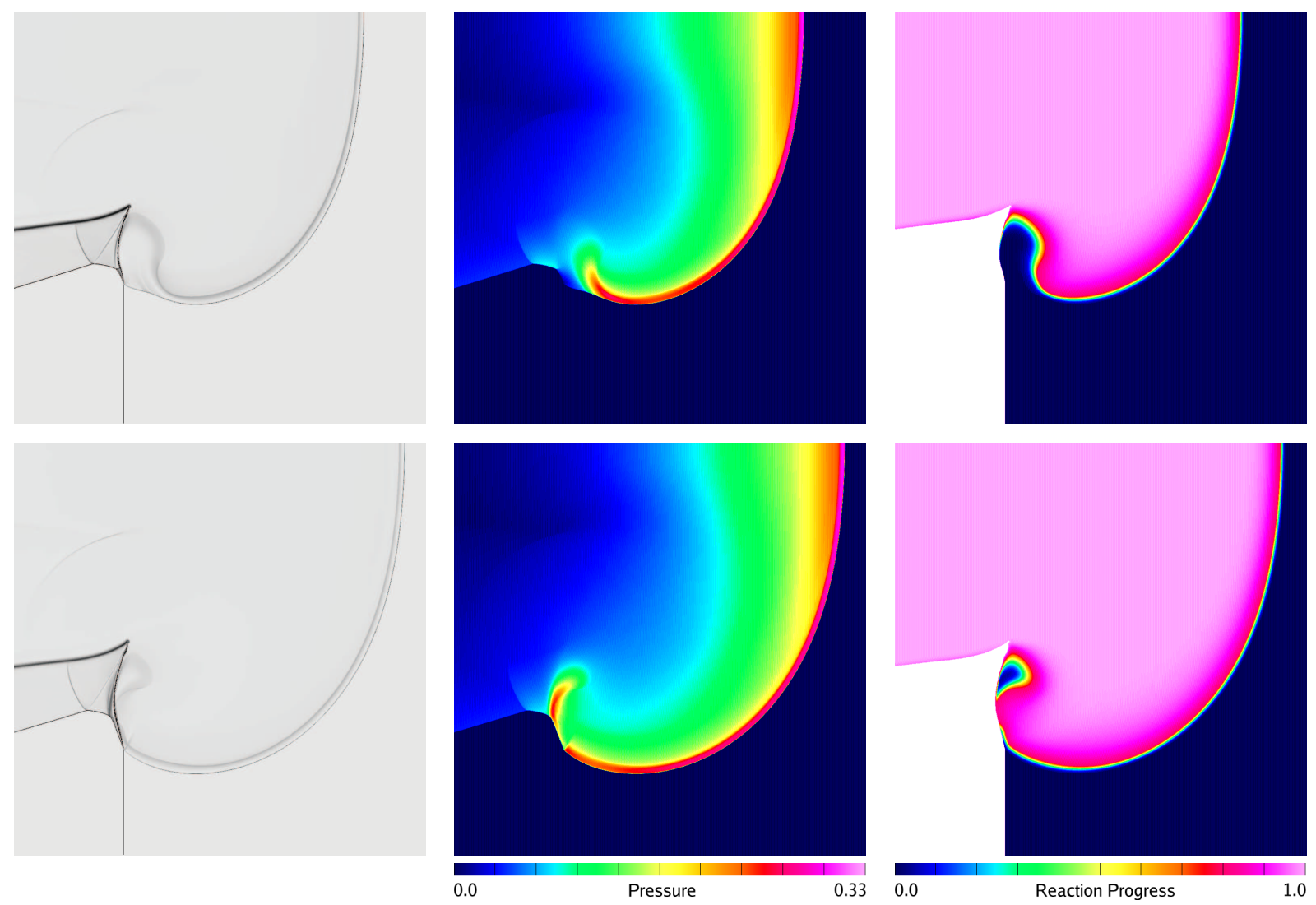

Figure 24: Weak confinement: schlieren (left), pressure (middle) and reaction progress (right) at $t=7.7$ (top frames) and $t=7.9$ (bottom frames).
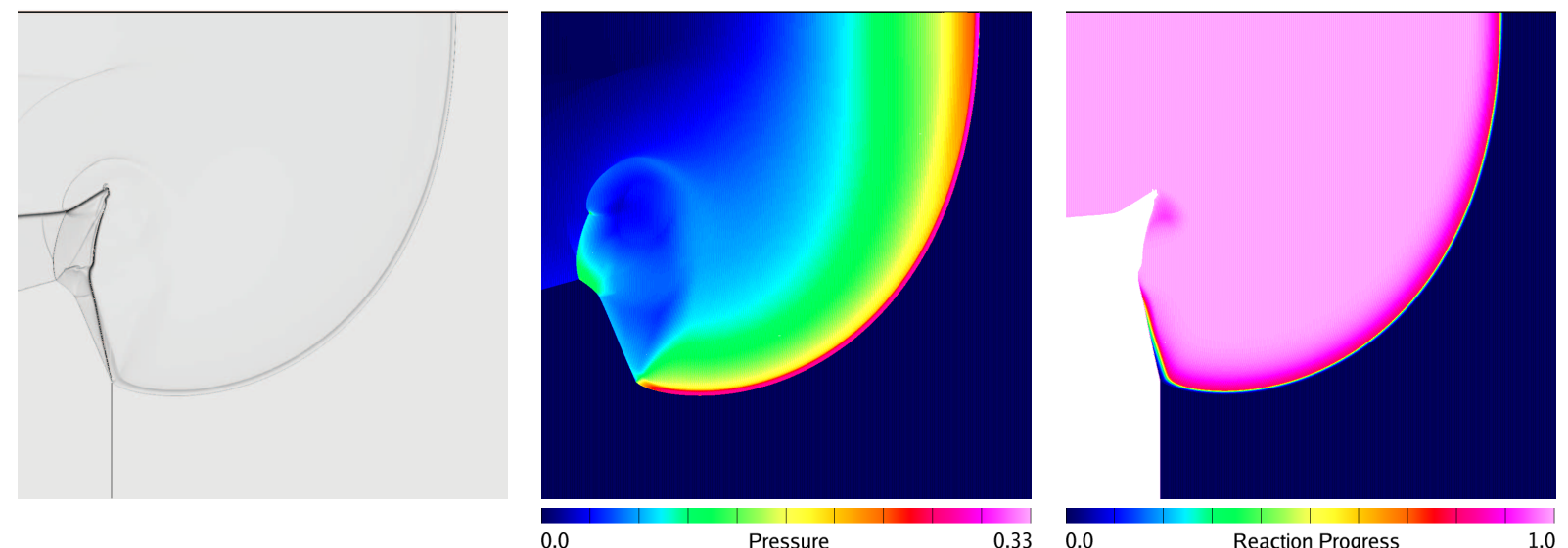

Figure 25: Weak confinement: schlieren (left), pressure (middle) and reaction progress (right) at $t=8.5$. 


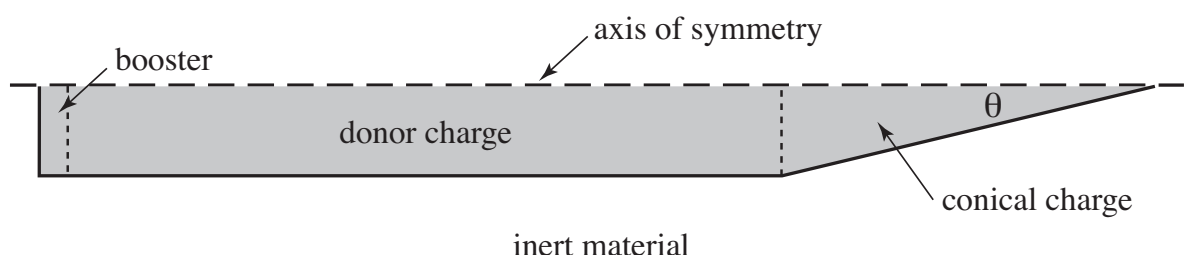

Figure 26: Schematic representation of the converging rate-stick geometry. The donor charge has a radius of 0.25 inches (equal to 0.8230 dimensionless units) and the included half-angle of the conical charge is $\theta$. A detonation is initiated on the left end of the weakly confined pencil-shaped charge by a high-pressure booster state.

the hockey-puck calculation in the previous study and the new weakly-confined corner-turning calculation here suggest that it is the geometry of the flow problem as well as the strength of the confinement that determine the diffraction behavior. If the diffraction is severe so that a significant separation of the leading shock and the reaction zone behind it occurs, then there appears to be strong tendency towards the formation of a secondary detonation and the subsequent consumption of the previously unreacted material in the solution of IG model so that no permanent dead zones occur. In contrast, the experiments in [10] for LX-17 and the ones in [13] for PBX-9502 both suggest that permanent dead zones do occur. One explanation for the difference is that the present IG rate law does not model desensitization due to weak-shock homogenization of the explosive material [14]. Thus, in the present weak-confinement calculation, for example, a temporary dead zone appears, but the unreacted material in it is not desensitized to subsequent reaction and thus it is ultimately consumed by a secondary reaction.

\subsection{Detonation propagation and failure in a conical rate stick}

The corner-turning problem described above considered detonation diffraction in an expanding geometry. In this subsection we study detonation propagation and dynamic failure in a converging geometry. Figure 26 provides an illustration of the geometry of the problem which is motivated by recent experiments performed by Salyer and Hill and discussed in [15]. A conical charge with an included half-angle $\theta$ is attached to a cylindrical donor charge with a 0.5 inch diameter. As in the previous problem, a detonation wave is initiated on the left end of the donor charge by a high-pressure booster state, and the axial length of the donor charge is long enough ( 2 inches in this case) so that the detonation achieves a steady state by the time it reaches the conical charge. The behavior of the reactive flow in the pencil-shaped explosive system is modeled using the governing equations in (4) with EOS described in Section 2.1 and rate law given in (13) for the IG model. The parameter for the EOS and rate law are taken to be those for PBX 9502 as given in tables 1 and 2, respectively. The explosive system is assumed to be weakly confined with EOS parameters given in table 1, which again is motivated by the experiments in [15].

The initial state of the flow in the explosive and in the inert is the same as that used for the corner-turning problem in Section 4.2, namely,

$$
\rho=1, \quad u_{1}=u_{2}=0, \quad p=0, \quad \lambda=0, \quad \phi_{r}=1,
$$

in the explosive, and

$$
\rho=1, \quad u_{1}=u_{2}=0, \quad p=0, \quad \lambda=0, \quad \phi_{r}=0,
$$

in the inert. In the booster state, the pressure and reaction progress are taken to be $p=0.24$ and $\lambda=1$, respectively, while the remaining variables in the explosive are unchanged. The calculations are carried out in two stages. The first stage involves the formation of the detonation and its propagation down the donor charge. This stage of the calculation is independent of the choice for the half-angle of the conical charge and thus can be done once for all values of $\theta$. By the end of this first stage, the detonation has reached a steady state and its behavior is shown in figure 27 . The numerical schlieren shows the undisturbed material interface 

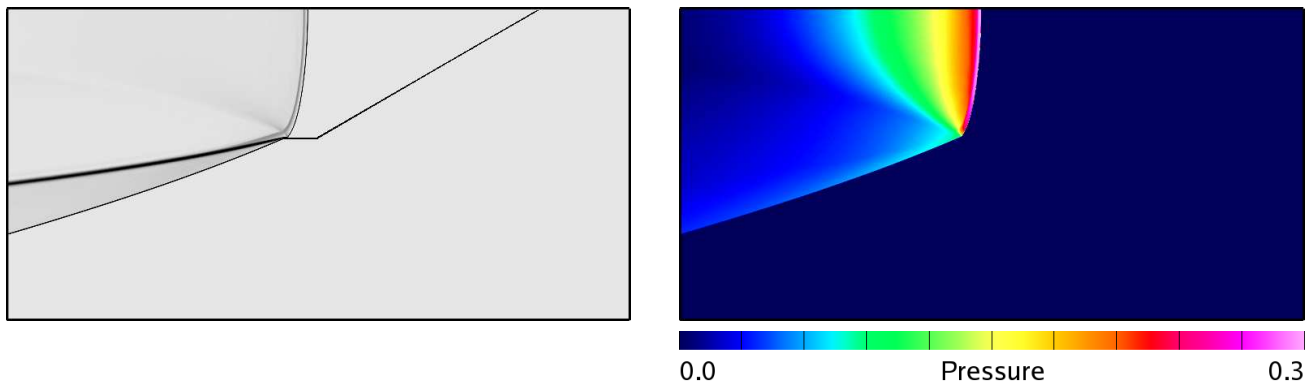

Figure 27: Numerical schlieren (left) and pressure (right) for a pencil-shaped explosive with $\theta=30^{\circ}$. The detonation has reached a steady state prior to its interaction with the conical charge, and this flow is used as an initial condition for the numerical calculation of the problem for any $\theta$.

ahead of the detonation as well as the deflected interface behind. There is a transmitted shock in the inert (as seen in all previous problems) and the detonation shock in the explosive is curved as a result of the release to the inert. The plot of pressure shows the high-pressure region immediately behind the detonation shock which is strongest along the axis of symmetry as expected. The second stage of the calculation begins with the state of the flow shown in figure 27 for all values of $\theta$, and involves the propagation of the detonation in the conical charge.

The main focus for this problem is dynamic failure of the detonation as it travels towards the tip of the conical charge for a chosen value of $\theta$. Figure 28 shows a schematic of the steady detonation structure in the donor charge with fixed radius $r_{0}$ just before it passes into the conical charge. The detonation shock at the material interface in the donor charge makes an angle $\xi_{\mathrm{s}}$ with the normal to the undisturbed interface corresponding to a sonic post-shock flow. This angle was determined earlier (see Section 4.2) using a shockpolar analysis and found to be $45.67^{\circ}$. At the moment when the detonation enters the conical charge, the angle between the detonation shock and the normal to the material interface increases abruptly and its new value corresponds to an oblique shock with a supersonic post-shock flow. As a result, acoustic information is unable to overtake the detonation shock so that no immediate adjustment is made at the interface to accommodate the change in the angle of the undisturbed material interface in the conical charge ahead of the shock. As the detonation travels down the conical charge, the angle between the detonation shock and the normal to the material interface decreases since the detonation flattens towards the axis of symmetry. If $\theta<\xi_{\mathrm{s}}$, then there is a radius, $r_{\mathrm{s}}$ say, within the conical charge where the angle between the detonation shock and the normal to the material interface takes the sonic value $\xi_{\mathrm{s}}$ again (see figure 28). Beyond this point, the shape of the detonation adjusts in an attempt to maintain $\xi=\xi_{\mathrm{s}}$ at the material interface, and the detonation may fail at some radius less than $r_{\mathrm{s}}$ as a result of the losses to the inert overtaking the decreasing energy production from reaction. Conversely, if $\theta>\xi_{\mathrm{s}}$, then the sonic point is not reached before the detonation meets the tip of the conical charge, and no failure occurs. Salyer and Hill refer to cones with $\theta<\xi_{\mathrm{s}}$ as subcritical while cones with $\theta>\xi_{\mathrm{s}}$ are labeled supercritical (see [15]).

We are interested in the behavior of the detonation as it propagates in subcritical conical charges with $\theta<\xi_{\mathrm{s}}=45.67^{\circ}$. We begin with the behavior for a relatively small value of $\theta$. Figure 29 shows numerical schlierens and shaded color contours of pressure at times $t=2.7,3.5$ and 4.3 for $\theta=10^{\circ}$. The sequence of schlieren plots show dynamic failure of the detonation as the reaction zone separates from the leading shock. The plots of pressure show the corresponding weakening of the leading shock during the failure process. The line plots in figure 30 provide further evidence of failure. These plots show the behavior of the density and pressure (solid curves) along the axis of symmetry and the behavior of the reaction progress (dashed curves) at various times during the process of failure. Distance along the axis of symmetry is measured relative to the left side of the conical charge (as indicated in the sketch in figure 28) and the tip of the cone is located at $x_{\mathrm{c}}=r_{0} \cot \theta=4.67$ for this value of $\theta$. The behavior of the density and pressure indicate a gradual weakening of the leading shock until a time between $t=4.3$ and $t=4.5$ when the post-shock density falls below the ignition threshold, $\rho_{\text {ign }}=1+a=1.214$, so that the ignition reaction turns off. Once this occurs, no reaction takes place in the remaining unreacted material. Near the material interface where the 


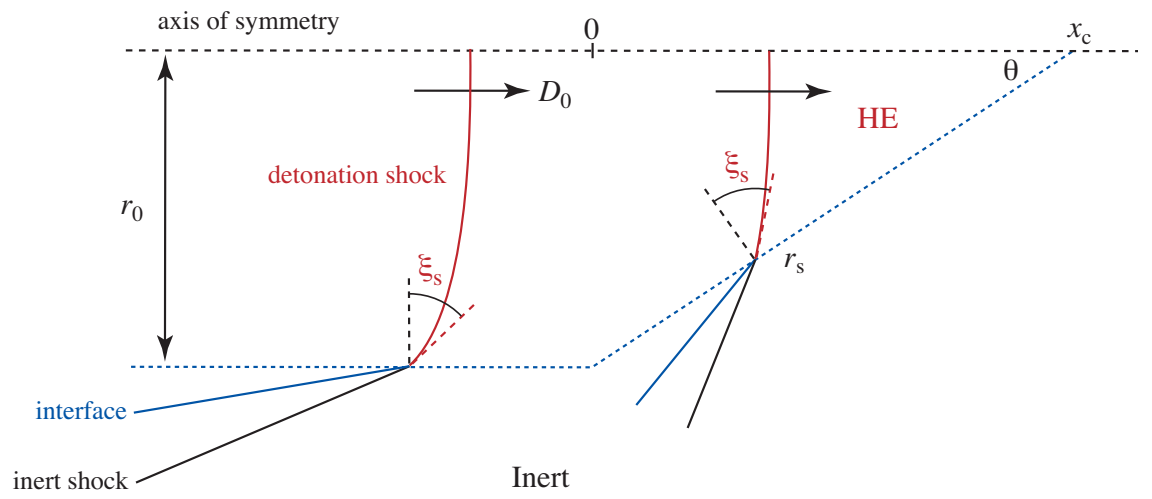

Figure 28: Schematic representation of the steady detonation shock with velocity $D_{0}$ in the donor charge with radius $r_{0}$ just prior to entering the conical charge. The angle between the detonation shock and the normal to the undisturbed material interface is $\xi_{\mathrm{s}}=45.67^{\circ}$ computed from a shock-polar analysis. At a later time, the detonation shock in the conical charge again makes an angle $\xi_{\mathrm{s}}$ at the material interface at radius $r_{\mathrm{s}}$.

detonation shock is weakest, in general, the ignition stage of the IG reaction rate fails sooner so that the failure process along the detonation profile occurs over a range of times. For reference, we note that an analysis of the steady detonation shock profile (taken from our numerical calculations) shows that the sonic angle $\xi_{\mathrm{s}}$ at the material interface is reached at $r_{\mathrm{s}}=0.81$ for this conical charge which is very close to the left side of the cone where $r=r_{0}=0.8230$. If we assume a nominal sound speed in the post-shock state to be that given by the sound speed in the von Neumann state of the corresponding planar detonation, then we can estimate the position along the axis of symmetry where an acoustic disturbance generated from $r=r_{\mathrm{s}}$ would first occur. This position, found to be $x_{1}=0.71$, provides a theoretical position for the earliest possible failure along the axis of symmetry. but our numerical calculations have shown that dynamic failure occurs much further towards the tip of the conical charge for $\theta=10^{\circ}$.

We now turn our attention to the behavior for a moderate value of $\theta$ in the subcritical range. Figure 31 shows a sequence of numerical schlierens and shaded contours of pressure for the case when $\theta=25^{\circ}$. For this value of $\theta$, failure occurs when the detonation has nearly reached the tip of the cone at $x_{\mathrm{c}}=1.76$, and so any separation of the reaction zone and the lead shock is difficult to see in these plots. The corresponding line plots showing the behavior of $\rho, p$ and $\lambda$ along the axis of symmetry for this case are provided in figure 32. Here, we see more clearly the weakening the detonation shock leading up to failure which occurs near $t=1.9$ when the post-shock density approaches $\rho_{\mathrm{ign}}$. The pressure peak behind the shock has reduced considerably by this time and there is a clear separation of the reaction zone as indicated by the behavior of $\lambda$ at $t=1.9$ in this plot. The value for $r_{\mathrm{s}}$ for $\theta=25^{\circ}$ is determined to be 0.75 . As before, we may estimate the corresponding position along the axis of symmetry where failure can first occur, and this is found to be $x_{1}=0.73$. Again this value only provides the leftmost position for detonation failure and the computed dynamic failure is found to occur much closer to the tip of the conical charge.

As a final example, we consider the behavior for a relatively large subcritical value of $\theta$. Figures 33 and 34 show the results for the case of $\theta=40^{\circ}$ which is fairly close to the sonic value $\xi_{\mathrm{s}}=45.67^{\circ}$. For this case, there is no apparent reduction in the post-shock pressure and no failure occurs. The line plots in figure 34 show essentially no change in the detonation structure along the axis of symmetry up to the tip of the conical charge at $x_{\mathrm{c}}=0.981$. The sonic radius for this case is determined to be $r_{\mathrm{s}}=0.38$ and the location along the axis of symmetry corresponding to the first acoustic response is $x_{1}=0.78$.

In the experiments performed by Salyer and Hill, the surface of the conical charge was polished so that the position of the detonation could be measured by an optical system that reflects light from the polished surface. As the detonation propagated down the conical charge, the polished surface was destroyed thus revealing the position of the wave at various times. We perform a similar analysis from our numerical calculations. In the undisturbed flow ahead of the detonation, the mass fraction of the reactive species, $\phi_{r}$, takes the 

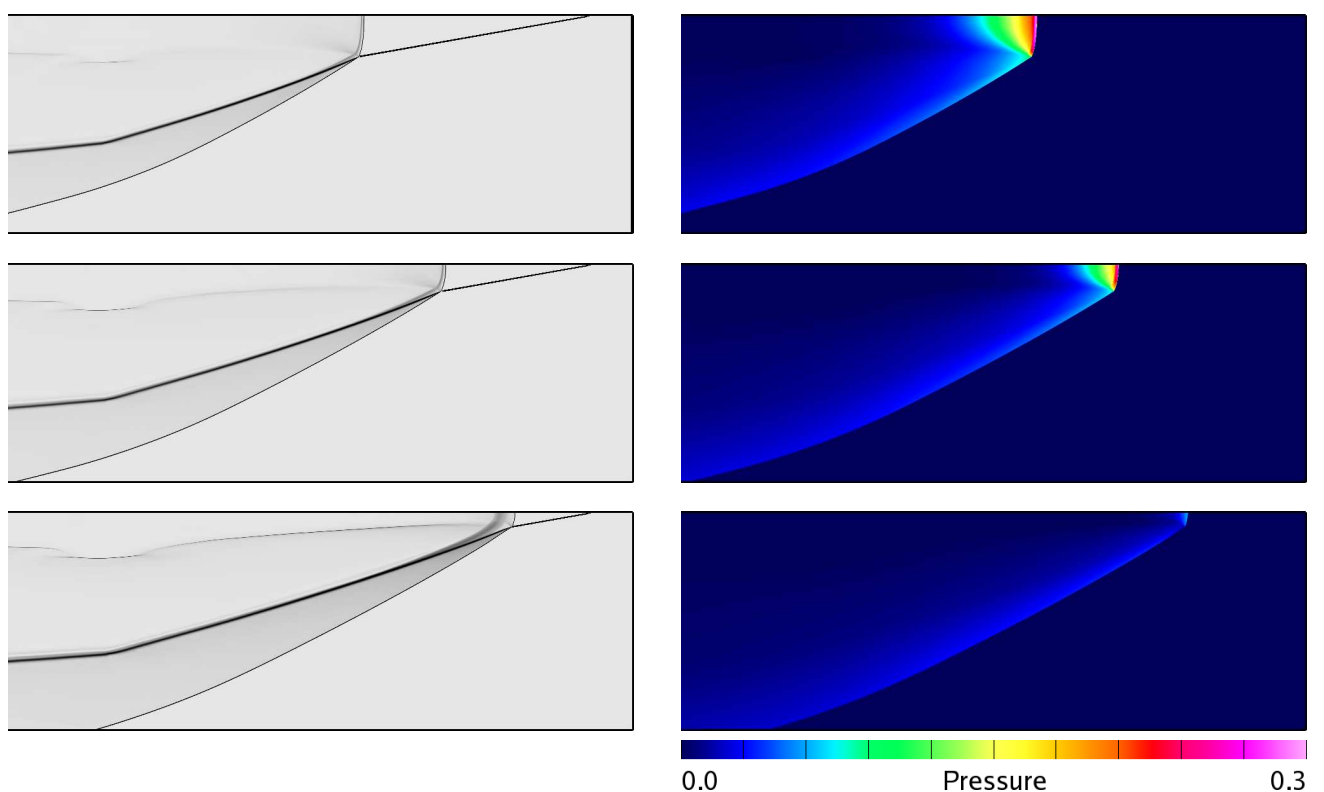

Figure 29: Numerical schlieren (left) and shaded contours of pressure (right) for the case of $\theta=10^{\circ}$ at $t=2.7$ (top), $t=3.5$ (middle) and $t=4.3$ (bottom).
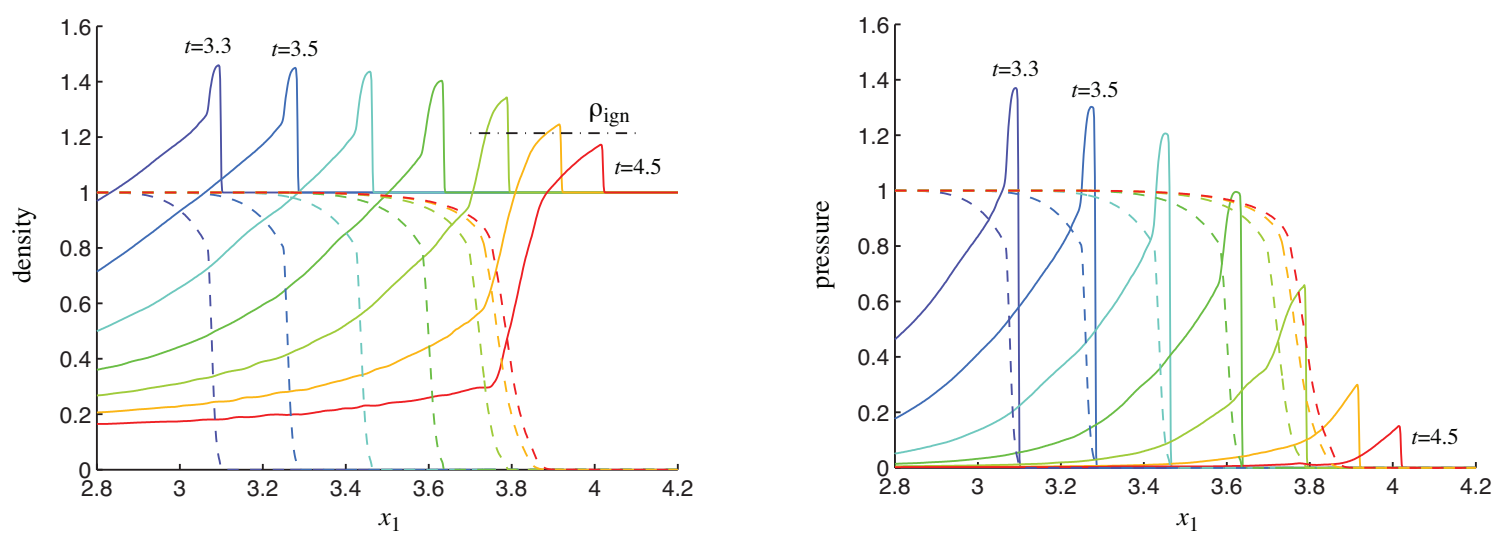

Figure 30: Behavior of density (left) and pressure (right) along the axis of symmetry for the case of $10^{\circ}$. The corresponding behavior of the reaction progress is shown as dashed curves in both plots, and the pressure is scaled by factor of 5 for plotting convenience. The ignition threshold $\rho_{\mathrm{ign}}=1+a=1.214$ is shown in the density plot. The cone tip is located at $x_{\mathrm{c}}=4.67$ 

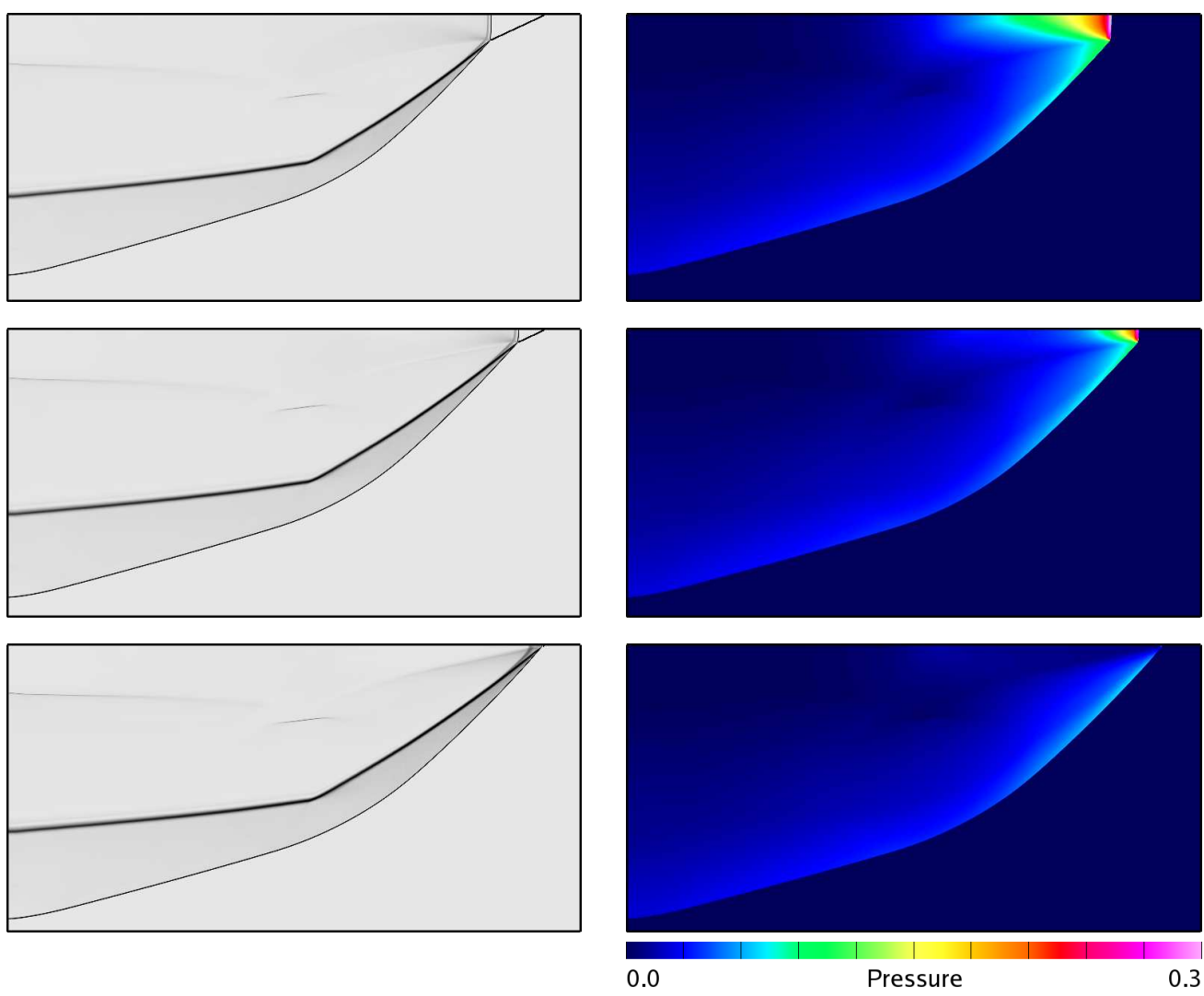

Figure 31: Numerical schlieren (left) and color contours of pressure (right) for the case of $\theta=25^{\circ}$ at $t=1.5$ (top), $t=1.7$ (middle) and $t=1.9$ (bottom).
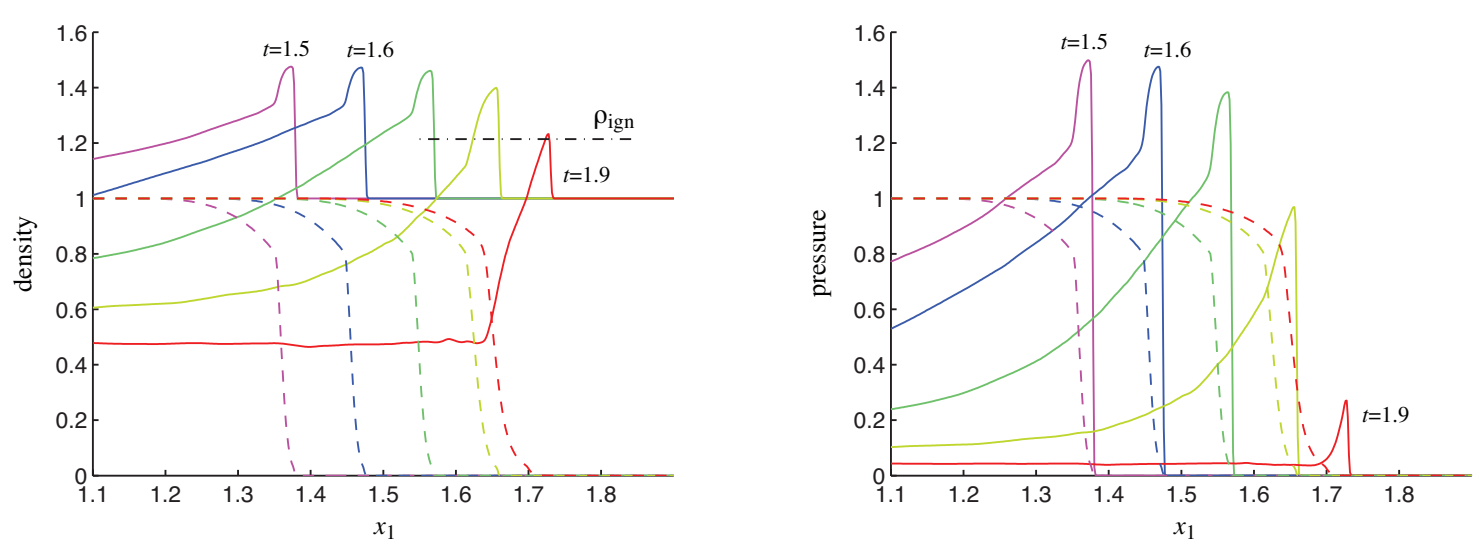

Figure 32: Behavior of density (left) and pressure (right) along the axis of symmetry for $25^{\circ}$. The corresponding behavior of the reaction progress is shown as dashed curves in both plots, and the pressure is scaled by factor of 5 . The ignition threshold $\rho_{\text {ign }}=1+a=1.214$ is shown in the density plot. The cone tip is located at $x_{\mathrm{c}}=1.76$ 

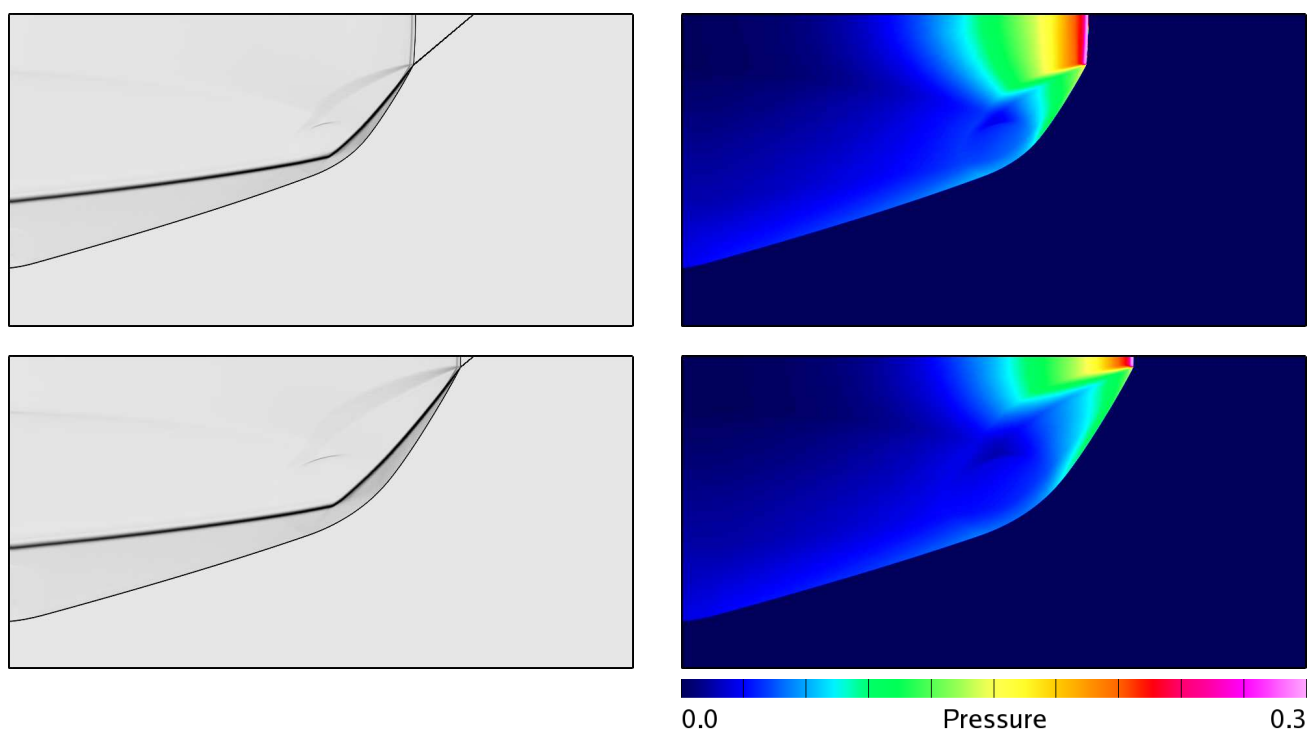

Figure 33: Numerical schlieren (left) and color contours of pressure (right) for the case of $\theta=40^{\circ}$ at $t=0.7$ (top) and $t=1.0$ (bottom).
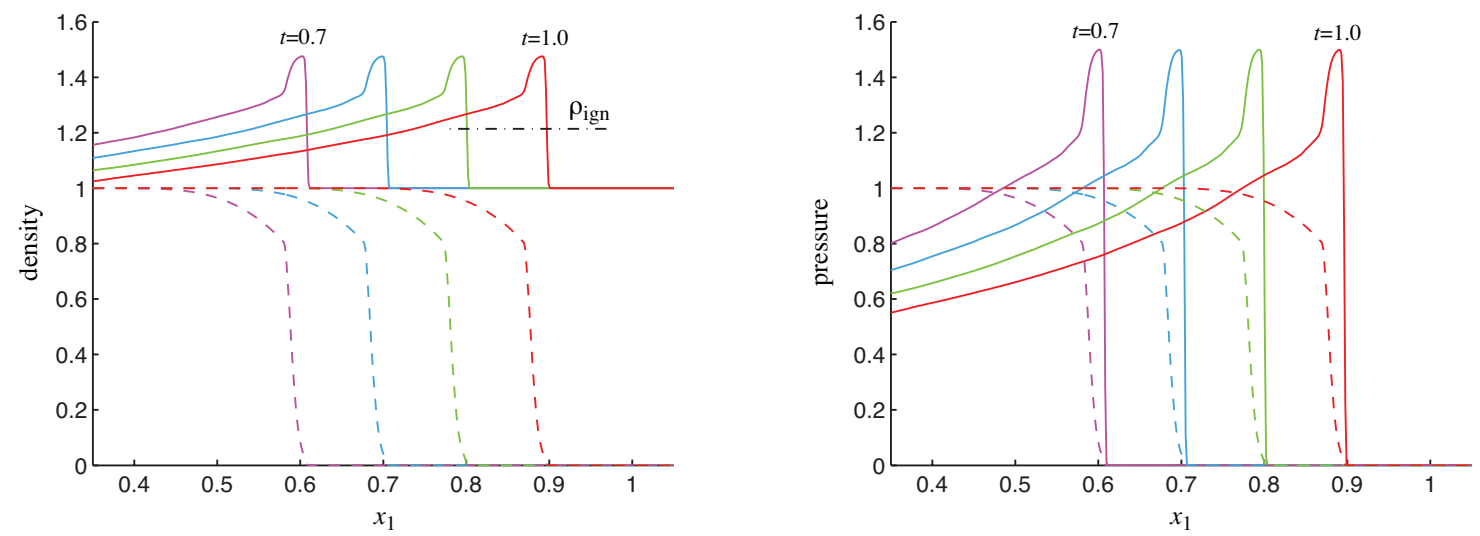

Figure 34: Behavior of density (left) and pressure (right) along the axis of symmetry for $40^{\circ}$. The corresponding behavior of the reaction progress is shown as dashed curves in both plots, and the pressure is scaled by factor of 5 . The ignition threshold $\rho_{\text {ign }}=1+a=1.214$ is shown in the density plot. The cone tip is located at $x_{\mathrm{c}}=0.981$ 

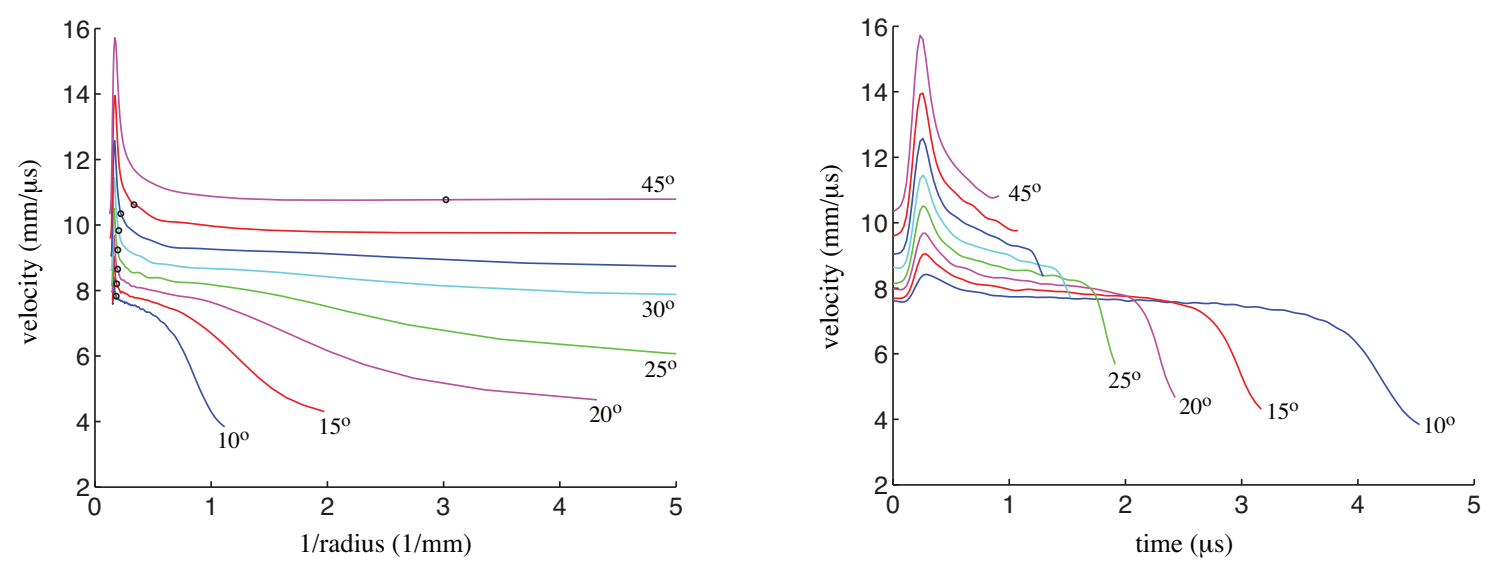

Figure 35: Surface velocity of the detonation versus inverse radius (left) and versus time (right) for conical changes with included half angles $\theta=10^{\circ}, 15^{\circ}, \ldots, 45^{\circ}$. Black circles mark $1 / r_{\mathrm{s}}$ for each value of $\theta$ in the left plot.

initial values 0 or 1 exactly, while in the disturbed flow behind the detonation the material interface is smeared so that $\phi_{r}$ takes values between 0 and 1 . We then measure the position of the detonation by choosing the largest value of $x_{1}$ for which $0.25 \leq \phi_{r} \leq 0.75$ at each time step. Using the position data from the experiments or the numerical calculations, the velocity of the detonation along the surface of the conical charge may be found. (In practice, some smoothing is required to eliminate high-frequency noise in the data.) Figure 35 shows a plot of the surface velocity of the detonation versus inverse radius and a plot of the surface velocity versus time determined from our numerical calculations for cone half-angles $\theta=10^{\circ}, 15^{\circ}, \ldots, 45^{\circ}$. Focusing first on the plot of surface velocity versus inverse radius, we see that after an initial jump in the velocity due to the oblique impact of the detonation with the conical material interface and the brief strengthening which occurs thereafter, the velocity settles to a value that is well-approximated by $D_{0} \sec \theta$, where $D_{0}$ is the steady detonation velocity in the donor charge (equal to 0.96 approximately). This is the surface velocity that would occur if the detonation made no significant adjustment from its steady velocity during the run down the conical charge. For the conical charges computed with $\theta$ between $10^{\circ}$ and $35^{\circ}$ the surface velocity of the detonation shows a decrease from the steady value, $D_{0} \sec \theta$, at some radius along the conical charge. The decrease, which is most apparent for the smaller values of $\theta$, indicates a failure of the detonation, and this failure occurs for $1 / r<1 / r_{\mathrm{s}}$ in each case. Upon failure the surface velocity decays rapidly to $c_{0} \sec \theta$, where $c_{0}$ is the ambient sound speed in the unburnt explosive, and again this is seen most clearly in the curves for smaller values of $\theta$. Salyer and Hill provide a similar plot in [15], and our plot is in good agreement with theirs indicating that the ignition-and-growth model performs well for this dynamic-failure problem.

We have also included a plot of surface velocity versus time in figure 35 because it shows the failure of the detonation more clearly. In this plot one sees the initial spike in velocity, as before, followed by a sharp decrease in the velocity for $\theta$ between $10^{\circ}$ and $35^{\circ}$ indicating failure of the detonation in those conical charges. The absence of failure for $\theta=40^{\circ}$ and $45^{\circ}$ is clear in this plot as the detonations for these two case run to the tip without any abrupt decrease in the surface velocity.

\section{Conclusions}

In this paper we have continued our previous study of the ignition-and-growth model, now extended to cases where the explosive is not rigidly confined, a situation closer to experimental setups. Two possible confinements are considered, weak and strong, so defined in terms of whether the the flow behind the lead shock in the vicinity of the explosive-confiner interface does, or does not, involve an expansion. Attention 
continues to be focused on the ability of the model to predict detonation failure, and the intent is to determine whether allowing the confiner to deform leads to a significant difference in model behavior.

From a computational viewpoint the presence of a deformable interface between the reactive material (either explosive or products of combustion) and the inert confiner introduces new challenges. It is known, for example, that Godunov schemes can generate spurious oscillations at the interface. This has required a significant enhancement of the numerical procedure that was used in the earlier rigid-wall IG calculations. In an earlier publication [21] we had developed an energy-correction procedure that captures the interface between two inert materials in a manner that is robust, accurate and free of numerical oscillations. Here we have extended that strategy to include the interface between a reactive and an inert material. The result is a computational tool that can be applied to hyperbolic, multi-material, reactive systems in two-dimensional and axisymmetric configurations.

The present study begins with the computation of detonation propagation in a two-dimensional rate stick. This oft-computed configuration is examined for two reasons: first, to introduce the notions of strong and weak confinement (following the work of [22]), and second, to establish convergence of the numerical solutions and confirm their accuracy by comparing with known analytic solutions. We then consider an axisymmetric configuration consisting of a donor stick of explosive expanding suddenly into an acceptor stick of larger radius. As the detonation turns the $90^{\circ}$ corner from the donor into the acceptor section, it undergoes diffraction, and experiments show the appearance of a dead zone at the corner. Computations show a different behavior, however. In the rigidly confined case we see that subsequent to corner turning, the reaction zone near the interface decouples from the lead shock but only temporarily. Preconditioning by the lead shock keeps the reaction at the interface alive; in due course the reaction accelerates, strengthens the lead shock, and the coupling revives the detonation. In the strongly confined case the small yielding of the interface produces a commensurately small modification of the evolution scenario. The slightly weaker reaction at the interface now takes somewhat longer to strengthen. The lead shock has advanced too far to remain directly coupled to the strengthening reaction, and the result is a local explosion occurring some distance behind the lead shock. This explosion produces a secondary shock of its own that overtakes the lead shock and the detonation is again re-established. In the weakly confined case the interface yields to a larger extent, thereby weakening the lead shock sufficiently so as to extinguish the initiation reaction near the interface. The result is the creation of a significant pocket of unreacted material in the vicinity of the interface behind the lead shock. Away from the interface there is an intermediate region behind the lead shock in which the reaction is alive and increasingly stronger, but too weak to be coupled to the lead shock. Still farther away from the interface the reaction and the lead shock are fully coupled in a detonation that never experienced diffraction. As the reaction in the intermediate region strengthens, it creates a curved pressure ridge, or hook, which is anchored to the undisturbed detonation at the far end. While the undisturbed detonation propagates forward into the pristine material with the primary shock as the lead shock, the hook transforms into a secondary detonation that propagates transversely towards the interface, thereby consuming the unreacted pocket. The upshot is that the compliant interface, whether strongly or weakly confining, does not materially influence the progression of post-diffraction events, in that sustained dead zones remain elusive for solutions of the IG model.

This study also examines detonation propagation and dynamic failure in a converging geometry. We find that when the curved detonation established in the straight section of the charge enters the conical section, its behavior depends upon the angle of the taper. For large cone angles the flow behind the detonation is supersonic, and the detonation does not sense the deformation of the explosive/confiner interface. As a result the detonation reaches the tip of the cone essentially at the same speed at which it entered the tapered portion. For small cone angles the following flow becomes sonic part way through the cone. The detonation then weakens; there is a significant decoupling and failure is indicated by a significant and precipitous decline in the speed of the wave. These computational results are entirely in accord with experiment.

We assert that the IG model must be extended to include explicitly the effect of desensitization by weak shocks if it is to replicate sustained failure and dead zones in the corner-turning configuration. Such an extension would account for diminution in the density of ignition sites due to compaction by a weak shock, and the associated increase in the strength of the stimulus needed for ignition. In the absence of such enhancement, computations have shown that even for compliant confinement, temporarily dormant regions created by the uncoupling of reaction from lead shock are subject to revival either on their own because they 
were weakly preconditioned by the lead shock and will ultimately explode, or because they can be swept by detonations generated elsewhere in the domain. In the cone such enhancement is not needed, and if included, will have little influence. This is because once the reaction zone and the lead shock have decoupled, the disturbances from the interface continue to weaken the reaction, and unlike the corner-turning case, no mechanism (self-strengthening or transverse wave) is available to re-establish the detonation.

\section{Acknowledgements}

Graduate research support for JWB was given by the National Science Foundation (NSF) under grants DMS9983646 and DMS-0312040, by the Office of Science, U.S. Department of Energy (DOE), through summer visits to the Lawrence Livermore National Laboratory (LLNL), and by Sandia National Laboratory under contract number DE-ACO4-94AL85000. Research support for DWS and AKK was given by NSF under grant DMS-0609874 and by Los Alamos National Laboratory under subcontract 23848-001-05. Additional research support for DWS was given by LLNL under subcontract B548468. Research supported for WDH was given under the auspices of the DOE by the University of California, LLNL under contract No.W-7405-Eng-48. The calculations in this paper were performed, in part, using the RPI Computational Math Cluster which was purchased using funds provided by NSF under grant DMS-0532160.

\section{References}

[1] E. L. Lee, C. M. Tarver, Phenomenological model of shock initiation in hetrogeneous explosives, Phys. Fluids 23 (12) (1980) 2362-2372.

[2] C. M. Tarver, J. O. Hallquist, Modelling two-dimensional shock initiation and detonation wave phenomena in PBX 9404 and LX-17, in: The Seventh Symposium (International) on Detonation, 1981, pp. $488-497$.

[3] C. M. Tarver, J. O. Hallquist, L. M. Erickson, Modelling short-pulse duration shock initiation of solid explosives, in: The Eighth Symposium (International) on Detonation, 1985, pp. 951-960.

[4] C. M. Tarver, J. W. Kury, R. D. Breithaupt, Detonation waves in triaminonitrobenzene, J. Appl. Phys. 82 (1997) 3771-3782.

[5] J. W. Kury, R. D. Breithaupt, C. M. Tarver, Detonation waves in trinitrotoluene, Shock Waves 9 (1999) $227-237$.

[6] C. M. Tarver, E. M. McGuire, Reactive flow modeling of the interaction of TATB detonation waves with inert materials, in: The Twelfth Symposium (International) on Detonation, 2002, pp. 641-649.

[7] M. R. Baer, J. W. Nunziato, A two-phase mixture theory for the deflagration-to-detonation transition (DDT) in reactive granular materials, Int. J. Multiphase Flow 12 (1986) 861-889.

[8] D. W. Schwendeman, C. W. Wahle, A. K. Kapila, The Riemann problem and a high-resolution Godunov method for a model of compressible two-phase flow, J. Comput. Phys. 212 (2006) 490-526.

[9] D. W. Schwendeman, C. W. Wahle, A. K. Kapila, A study of detonation evolution and structure for a model of compressible two-phase reactive flow, Combust. Theory and Modeling (submitted).

[10] C. M. Tarver, Ignition-and-growth modeling of LX-17 hockey puck experiments, Propellants, Explosives and Pyrotechnics 30 (2005) 109-117.

[11] A. K. Kapila, D. W. Schwendeman, J. B. Bdzil, W. D. Henshaw, A study of detonation diffraction in the ignition-and-growth model, Combust. Theory and Modeling (accepted).

[12] W. D. Henshaw, D. W. Schwendeman, An adaptive numerical scheme for high-speed reactive flow on overlapping grids, J. Comput. Phys. 191 (2) (2003) 420-447. 
[13] E. Ferm, C. Morris, J. Quintana, P. Pazuchanic, H. Stacy, J. Zumbro, G. Hogan, N. King, Proton radiography examination of unburned regions in PBX 9502 corner turning experiments, Tech. Rep. LA-UR-01-3555, Los Alamos National Laboratory (2001).

[14] G. de Olivera, A. K. Kapila, D. W. Schwendeman, J. B. Bdzil, W. D. Henshaw, C. M. Tarver, Detonation diffraction, dead zones, and the ignition-and-growth model, in: The Thirteenth Symposium (International) on Detonation, 2006.

[15] T. R. Salyer, L. G. Hill, The dynamics of detonation failure in conical PBX 9502 charges, in: The Thirteenth Symposium (International) on Detonation, 2006.

[16] S. Karni, Multicomponent flow calculations by a consistent primative algorithm, J. Comput. Phys. 112 (1994) 31-43.

[17] J. J. Quirk, S. Karni, On the dynamics of a shock-bubble interaction, J. Fluid Mech. 318 (1996) 129-163.

[18] R. Abgrall, How to prevent pressure oscillations in mulicomponent flow calculations: A quasi conservative approach, J. Comput. Phys. 125 (1996) 150-160.

[19] P. Jenny, B. Müller, H. Thomann, correction of conservative Euler solvers for gas mixtures, J. Comput. Phys. 132 (1997) 91-107.

[20] R. Saurel, R. Abgrall, A simple method for compressible multifluid flows, SIAM J. Sci. Comput. 21 (3) (1999) 1115-1145.

[21] J. B. Banks, D. W. Schwendeman, A. K. Kapila, W. D. Henshaw, A high-resolution Godunov method for multi-material flows on overlapping grids, J. Comput. Physics 223 (2007) 262-297.

[22] T. D. Aslam, J. B. Bdzil, Numerical and theoretical investigations on detonation-inert confinement interactions, in: The Twelfth Symposium (International) on Detonation, 2002, pp. 483-488.

[23] W. Fickett, W. C. Davis, Detonation, University of California Press, Berkeley, 1979.

[24] B. M. Dobratz, Properties of chemical explosives and explosive simulants, Tech. Rep. UCRL-51319, Lawrence Livermore Laboratory Report (1974).

[25] M. Short, I. I. Anguelova, T. Aslam, J. B. Bdzil, A. K. Henrick, G. J. Sharpe, Stability of idealized condensed phase detonations, preprint.

[26] G. Chesshire, W. Henshaw, A scheme for conservative interpolation on overlapping grids, SIAM J. Sci. Comput. 15 (4) (1994) 819-845.

[27] M. Berger, On conservation at grid interfaces, SIAM J. Numer. Anal. 24 (5) (1987) 967-984.

[28] M. J. Berger, J. Oliger, Adaptive mesh refinement for hyperbolic partial differential equations, J. Comp. Phys. 53 (1984) 484-512.

[29] W. D. Henshaw, D. W. Schwendeman, Moving overlapping grids with adaptive mesh refinement for high-speed flow, J. Comput. Phys. 216 (2) (2006) 744-779. 